Article

\title{
Multi-Hop-Enabled Energy-Efficient MAC Protocol for Underwater Acoustic Sensor Networks
}

\author{
Khaja Shazzad, Kemal Tepe * and Esam Abdel-Raheem \\ Department of Electrical and Computer Engineering, University of Windsor, Windsor, \\ ON N9B 3P4, Canada; E-Mails: shazzad@uwindsor.ca (K.S.); eraheem@uwindsor.ca (E.A.-R.) \\ * Author to whom correspondence should be addressed; E-Mail: ktepe @uwindsor.ca; \\ Tel.: +1-519-253-3000 (ext. 3426).
}

Academic Editors: Jaime Lloret Mauri and Sandra Sendra

Received: 1 January 2015 / Accepted: 7 August 2015 / Published: 2 September 2015

\begin{abstract}
In multi-hop underwater acoustic sensor networks (UWASNs), packet collisions due to hidden and local nodes adversely affect throughput, energy efficiency and end-to-end delay. Existing medium access control (MAC) protocols try to solve the problem by utilizing a single-phase contention resolution mechanism, which causes a large number of control packet exchanges and energy overhead. In this paper, we introduce a MAC protocol that splits this single-phase contention resolution mechanism into two phases to provide efficient multi-hop networking. In the first phase, local nodes are eliminated from the contention, and in the later phase, the adverse effects of hidden nodes are mitigated. This two-phased contention resolution provides higher energy efficiency, better throughput and shorter end-to-end delay, and it also enables adaptability for different network architectures. A probabilistic model of the proposed protocol is also developed to analyse the performance. The proposed protocol has been evaluated through quantitative analysis and simulation. Results obtained through quantitative analysis and simulation reveal that the proposed protocol achieves significantly better energy efficiency, higher and more stable throughput and lower end-to-end delay compared to existing protocols, namely T-Lohi and slotted floor acquisition multiple access (S-FAMA).
\end{abstract}

Keywords: MAC protocols for underwater sensor networks; underwater acoustic communication; MAC protocol; sensor network; multi-hop network 


\section{Introduction}

The first underwater acoustic (UWA) communication was realized between submarines during World War II [1]. Since then, the need for underwater communication systems and networking has steadily increased for various crucial applications, such as the exploration of underwater resources, preventing and forecasting natural and man-made disasters, monitoring underwater environmental and marine habitats and military and commercial surveillance [2-6]. Most of the systems deployed for these applications perform an off-line operation, where there is a large latency between the collection and the utilization of data. In order to increase the efficiency and effectiveness for underwater communication systems, a standard similar to IEEE 802.11 is required [7], which has paved the way for explosive growth in wireless networking and enabled several applications. A similar standard in UWA networks provides growth by allowing reusable commonly-available hardware and software and cost-effective plug-and-play components for rapid deployment. One of the stepping stones in providing such a standard for UWA networks is to design a medium access control (MAC) protocol allowing efficient and effective multi-hop networking, which is the main focus of this paper.

UWA communication has its own unique challenges. First, propagation delay in a UWA channel is very large due to the low speed of the sound in the water, which is $1500 \mathrm{~m} / \mathrm{s}$, compared to the speed of radio waves, which is $3 \times 10^{8} \mathrm{~m} / \mathrm{s}$. This low propagation delay does not allow the utilization of carrier sense multiple access (CSMA) schemes [8]. Moreover, UWA is highly non-symmetric from an energy usage perspective for transmission and reception, where the transmission energy consumption is approximately one hundred times more than the reception energy consumption [9]. Consequently, packet collisions not only reduce the channel utilization, but also consume precious energy resources. One of the recommended ways to increase energy efficiency in UWA communication is to utilize multi-hop mesh network technology [10]. That is why there is an emphasis on designing a multi-hop-ready MAC protocol for UWA channels for energy efficiency operation.

The two important metrics in the design of a MAC layer protocol for a multi-hop underwater acoustic sensor network (UWASN) are to minimize packet collisions (for both control and data) and to increase energy efficiency. Similar to fully-connected wireless communication systems, frame collisions in UWASNs are mainly caused by local and hidden nodes. Channel reservation using control packets is one of the techniques that has been utilized by the existing MAC solutions for UWASNs. The energy consumption required for those control packet exchanges is very high, which is not suitable for UWASNs. Therefore, tone-based contention schemes, like T-Lohi, were proposed [9]. T-Lohi tries to resolve contention in a single-hop UWASN; however, it does not perform well in multi-hop scenarios where mesh networking is employed. In the MAC proposal presented in this paper, contention resolution is performed utilizing tones in the single-hop contention region in the first phase, and then, request to send (RTS) and clear to send (CTS) reservations are employed for multi-hop communication in the second phase. This two-phased contention resolution reduces energy consumption, packet collisions and delay significantly and allows stable operation in multi-hop UWASNs.

In this paper, the proposed MAC protocol will be described, and a probabilistic analysis will be carried out, where stability and theoretical performance results will be derived. Then, network simulations are performed to evaluate the proposed protocol and are compared to two prominent UWASN MAC 
protocols. Those comprehensive simulation studies verify the results derived out of probabilistic analysis and show that the designed MAC provides more stable and better performance in multi-hop UWA network scenarios. The rest of the paper is organized as follows. In Section 2, we discuss the related works. We present and describe our proposed protocol in Section 3. Section 4 presents the quantitative analysis and performance evaluation of the proposed protocol. In Section 5, simulation results are demonstrated, while conclusions are provided in Section 6.

\section{Related Works}

UWASNs play an important role to understand and monitor the largest resources on our planet, oceans, seas and lakes, and to maintain those resources in healthy and sustainable conditions. That is why UWA sensor networking activities are progressively increasing. MAC protocols are very important and are considered the challenging part of the UWASNs to provide contention-free and efficient networking. This section provides a brief overview of related existing MAC protocols for UWASNs.

Since energy efficiency is very important for UWASNs, Rodoplu et al. proposed an energy-efficient MAC protocol (EE-MAC) for highly-dense, short-range and fully-connected UWASNs [11], which introduces a very low duty cycle similar to the S-MAC [12] proposed for wireless sensor networks (WSN). The main contribution of EE-MAC is to provide energy efficiency by minimizing the idle listening period. The protocol assumes time-invariant propagation delay between any pair of nodes in the network and a large transmission cycle compared to the maximum propagation delay. EE-MAC reduces the energy loss due to packet collisions; however, the throughput of the system is considered low and, hence, cannot accommodate large data dissemination applications. Another disadvantage of EE-MAC [11] is that it is not suitable for multi-hop networks, because it does not provide any collision avoidance mechanism, which is crucial in multi-hop networks.

In [8], it was shown that due to the large propagation delay in UWASN, slotted ALOHA (S-ALOHA) performs similar to pure ALOHA with a guard time equal to the maximum propagation delay of the network. In [13], ALOHA was studied in multi-hop UWASN for string topology (i.e., a chain). In this paper, the analysis technique introduced in [13] is modified and enhanced in order to model and analyse the proposed protocol.

The slotted floor acquisition multiple access (S-FAMA) protocol for UWASN was proposed in [14]. It extends the FAMA non-persistent carrier sensing (FAMA-NCS) protocol proposed in [15] to consider terrestrial wireless networks, since terrestrial wireless channels and UWA channels have relatively large propagation delay. In the original FAMA [16], the channel is acquired by a potential transmitting station prior to any data transmission using RTS and CTS messages as in the collision avoidance technique proposed by multiple access collision avoidance for wireless LAN (MACAW) in [17] after a brief period of carrier sensing. FAMA-NCS enhanced the original FAMA using long RTS and CTS control packets to ensure collision-free data transmission in multi-hop networks with large propagation delay. S-FAMA eliminates the need for long RTS and longer CTS required in FAMA-NCS by slotting and synchronizing frames and constraining the transmission of both control and data packets at the beginning of a slot.

S-FAMA- and MACAW-based MAC protocols eliminate collisions caused by local and hidden nodes through a single RTS/CTS cycle, which can be called a monolithic reservation, as RTS and CTS control 
packets are used to eliminate both local and hidden nodes simultaneously from channel contention in a single reservation period. However, in the proposed MAC, the channel reservation mechanism is divided into two different phases. In the first phase, local contending nodes are eliminated using simple tone exchanges, then in the next phase, RTS and CTS are utilized to reserve the channel only to eliminate collision due to contending hidden nodes. This two-phased reservation provides several performance and design benefits, namely: energy savings due to both low power tone utilization instead of control packets and collision avoidance from hidden nodes, higher channel utilization, lower effective end-to-end delay, modular architecture and ease of implementation, adaptation and reconfiguration depending on the traffic types, loads and network topology.

The T-Lohi MAC protocol was proposed for UWASN in [9], and it is considered one of the first MAC protocols that suggested the use of tones to reserve the channel. Although T-Lohi is efficient and effective as a MAC, it does not solve the hidden station problem. Because of that, T-Lohi does not perform well in multi-hop networking. Another interesting suggestion made by T-Lohi was to utilize two receivers (one as a wake-up tone receiver and another as a data receiver) and one transmitter. The advantage of using two different receivers reduces the energy consumption during idle listening. The micro-modem proposed by Woods Hole Oceanographic Institution (WHOI) [18] is a small-footprint, low-power acoustic modem based on the Texas Instruments TMS320C5416 DSP. It has the capability to be used as a low power tone receiver, as well as a data receiver.

Recently, a number of TDMA-based MAC protocols have been introduced in the literature. In [19], a TDMA-based MAC protocol, named efficient communication scheduling (ECS), was introduced with continuous time slicing. ECS shows improvement in channel utilization compared to traditional slotted TDMA in some specific topologies. In [20], Lee et al. proposed a multi-hop reservation MAC protocol where a single reservation can be used for a multi-hop multiple packet transmission and provides a better end-to-end delay and throughput for uni-directional data dissemination, but experiences higher implementation complexity unfairness and longer reservation time for bi-directional data dissemination. In [21], Noh et al. proposed a delay-aware MAC protocol algorithm in which the propagation delays of the neighbouring nodes are locally calculated using the time-stamp information embedded in RTS packets. This allows an estimated expected transmission time of the packet for collision-free reception by the receiving node to be calculated. The algorithm also provides concurrent transmission and reception, exploiting temporal and spatial differences among the neighbouring nodes.

\section{Proposed MAC Protocol Design}

Let us consider a typical multi-hop network scenario as depicted in Figure 1. In any scenario, two node sets, $s=\left\{S_{0}, S_{1}, S_{2}\right\}$ and $h=\left\{H_{0}, H_{1}, H_{2}\right\}$, are considered out of interference range of each other; however, they are in the range of a possible receiving or relaying node, $r=\{R\}$. Here, the source node belongs to $s$ and $h$ sets, and $r$ is the receiver.

Any two nodes, $S_{i}, S_{j} \in s$ and $H_{k}, H_{l} \in h$, where $i \neq j$ and $k \neq l$, are connected to each other by only a single hop. Sets $s$ and $h$ are treated as local collision domains to $S_{i}$ and $H_{i}$, respectively. Each node in the sets $s$ and $h$ can contend for the channel independently with each other using any channel reservation or contention resolution technique. In a point of time, a single node from each set (assume $S_{0}$ from $s$ and 
$H_{0}$ from $h$ ) would have won the contention and reserved the channel to send a data packet to $R$. $S_{0}$ and $H_{0}$ do not have any information about the state of the other. Thus, if they send a data packet to $R$, two data packets arrive at $R$ at the same time, which causes a collision. Here, node sets $s$ and $h$ are hidden from each other and form two different independent collision domains. Each node in the sets $s$ and $h$ considers the other one as a hidden collision domain.

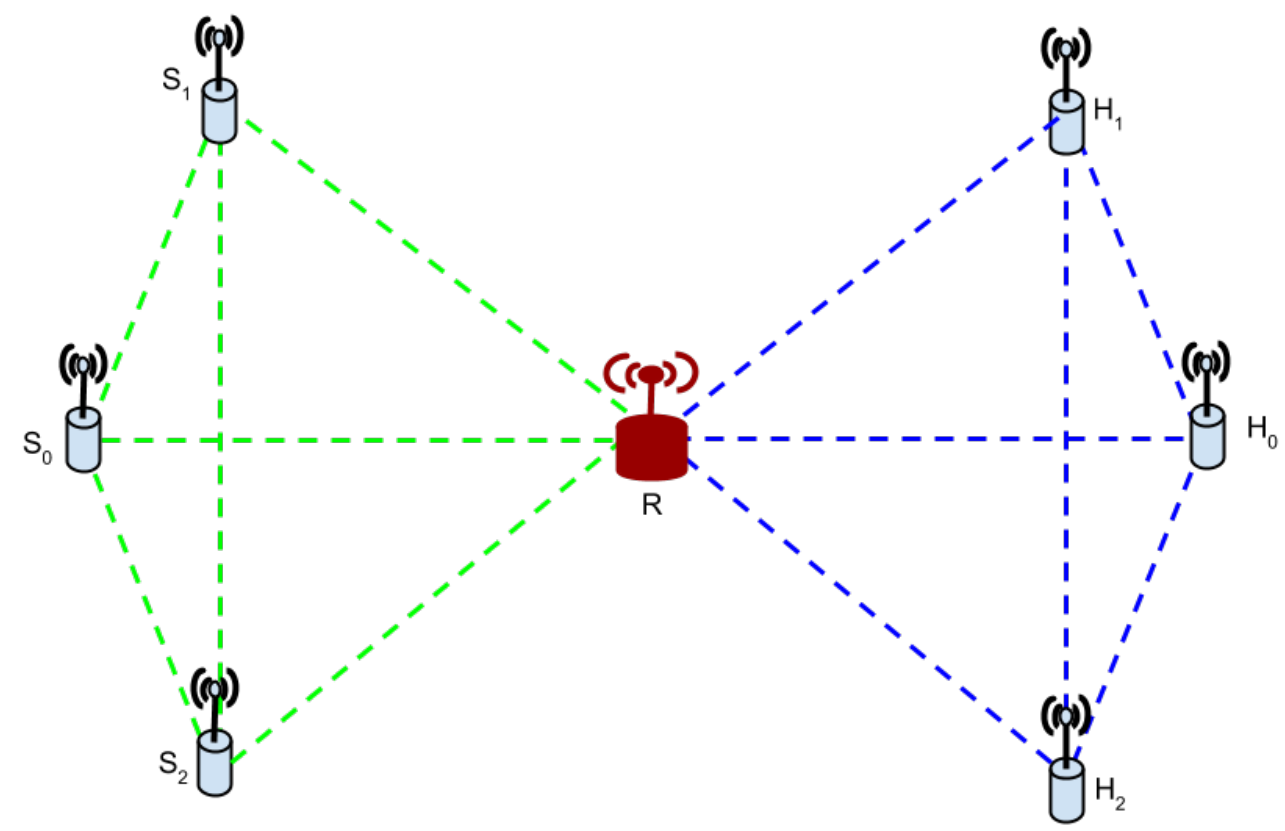

Figure 1. Collision domains in a wireless network.

Therefore, it is evident that in a multi-hop network, successful collision avoidance depends on two distinct collision domains, i.e., local and hidden collision domains. The MAC protocol design philosophy presented in this paper is to separate the channel contention resolution into two phases. In Phase 1, data packet collisions due to local collision domains are avoided through local channel reservation. This phase is named "local link reservation". In Phase 2, data packet collisions due to hidden collision domains are avoided through controlling the corresponding hidden channels. This phase is named the "hidden link control" phase.

In the following two sub-sections, we will describe the protocol, the impact and advantages of the hidden link control mechanism in a multi-hop network scenario.

\subsection{Protocol Description}

In the proposed protocol, a successful data transmission implies that the data is successfully received by the intended receiver or receivers. Successful data transmission and reception is visualized by a logical time frame of variable length, which consists of three parts, as shown in Figure 2:

1. Local link reservation;

2. Hidden link control;

3. Data transmission. 
A time frame consists of multiple time slots. Local link reservation and hidden link control phases consist of one or more time slots. Each time slot is called a contention round (CR). The duration of the $\mathrm{CR}$ is determined such that any tone or transmitted control packet must be received by all of the nodes in a local collision domain $[9,14,15]$. Short duration tones are utilized to resolve the contention among the nodes in the local link reservation phase. In order to reduce the energy lost due to idle listening, two different types of receivers are used: a low power wake-up tone receiver [18] and a data receiver. The data receiver is kept in sleep mode most of the time and is turned on by the wake-up tone receiver when needed. Therefore, a data packet always follows a wake-up tone. All nodes in the network are assumed to be synchronized. Transmission of each node must start at the beginning of a slot. The minimum duration of a slot must be equal to the maximum propagation delay between any pair of nodes in the network plus the time duration required to detect any wake-up tone by the receiver.

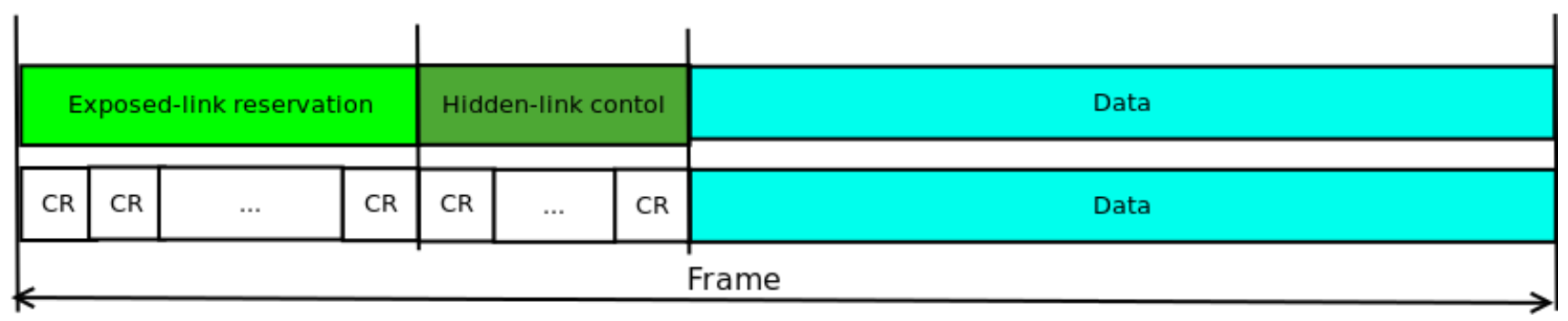

Figure 2. Successful data transmission frame structure for the proposed protocol.

In the local link reservation phase, short tones are used to resolve contention among the nodes. When the MAC layer receives a packet from an upper layer, it queues the packet and starts the local link reservation. At the beginning of a frame, the node starts contending for the channel by transmitting a tone. The contending node switches its modem to listening mode as soon as the transmission of the tone ends and starts listening to the channel until the current slot duration expires. This completes a single contention round. During this period, if the contending node does not receive any tone, the local link reservation is successful, and the hidden link control phase can be started. The reception of at least one tone in a CR manifests that there are other nodes that also want to transmit. In this case, the contending node increases its counter by the number of received tones and at the beginning of the next slot, it enters into a random back-off period, which is uniformly distributed between zero and the number of contending nodes multiplied by CR. When the back-off timer expires, the node contends again until it can reserve the channel. A single reservation period (RP) is required for local link reservation, and it consists of one or more CRs. After the local link reservation, the hidden link control phase starts. This local link reservation phase is similar to the channel reservation technique used in [9]. During local link reservation, any non-contending node that received a tone while in back-off state enters into a quiet state (i.e., it yields to other nodes to transmit data).

In order to eliminate collisions due to hidden collision domains, local link reservation is supported by the hidden link control phase. Hidden link control is carried out by the exchange of two distinct control packets prior to a data transmission. These additional control packets ensure that the nodes connected to the destination by hidden links defer their transmissions while the destination is receiving the data packet from its local link. The additional control packets are RTS and CTS. After the local link reservation, the sender broadcasts RTS and waits for one more slot to receive the CTS from the receiver. If the sender 
successfully receives a CTS from the receiver, it sends its data packet in the next slot, and thus, collisions are avoided. If the sender does not receive CTS from the receiver within the next slot duration, it backs off and invalidates its local link reservation. Nodes within the interference range of the sender receive an RTS packet and go to sleep mode until the end of the next slot, thus conserving energy. Nodes within the interference range of the destination receive a CTS and also go to sleep mode until the end of the transmission between the sender and the receiver. A node receiving more than one successful RTS packet in a given slot prioritizes those packets and selects one node to be the potential sender. This is done by setting the destination field address as the potential sender's address.

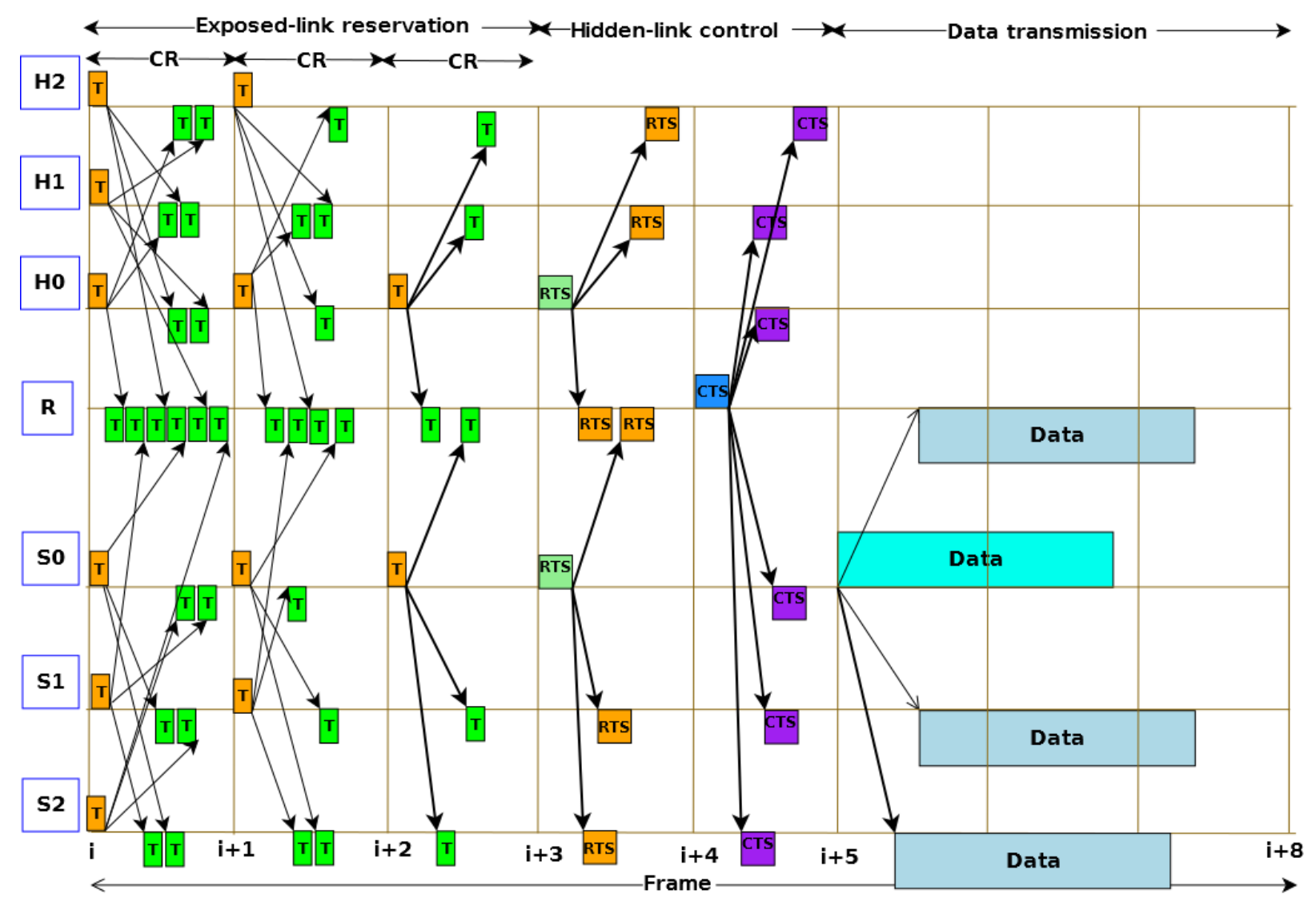

Figure 3. Medium access control (MAC) mechanism of the proposed protocol.

A typical successful data transmission (which is represented by a logical time frame) scenario is depicted in Figure 3 for a typical network scenario shown in Figure 1. Assume that each node of the collision domains wants to send the data packet to the receiving node $R$. At the beginning of a frame ( $i$-th slot), nodes $S_{0}, S_{1}$ and $S_{2}$ of the collision domain $s$ and nodes $H_{0}, H_{1}$ and $H_{2}$ of the collision domain $h$ start contention for local link reservation by transmitting tones. After transmission of the tone, each contending node switches to listening mode to detect the tones from neighbours. By the end of the slot, $S_{0}, S_{1}$ and $S_{2}$ nodes would have recorded the number of contending nodes (numberOfContendingNodes), which is three in this scenario. Similarly, contention among $H_{0}, H_{1}$ and $H_{2}$ is resolved by tone, also, since each contending node from both collision domains experiences more than one contender, each node switches its state to the back-off mode and the duration of the back-off mode is equal to a random variable uniformly distributed on the interval [0, numberOfContendingNodes) slots. Assume that nodes $S_{0}, S_{1}, H_{0}$ and $H_{2}$ select zero back-off 
slots and $S_{2}$ and $H_{1}$ select back-off Slots 1 and 2, respectively. Therefore, $S_{0}, S_{1}, H_{0}$ and $H_{2}$ send another tone at the beginning of the $(i+1)$-th slot, while $S_{2}$ and $H_{1}$ are both in back-off mode. By the end of the slot, each contending node has found that the number of contending nodes are now two. Like the previous slot, now the contending nodes back-off by selecting a uniformly-distributed random number between [0,2] slots. Assume that nodes $S_{0}$ and $H_{0}$ select zero back-off slots and others get non-zero back-off slots. Nodes $S_{0}$ and $H_{0}$ send another tone at the beginning of the $(i+2)$-th slot and start listening for the tones for the rest of the slot. By the end of this slot, contending nodes $\left(S_{0}\right.$ and $\left.H_{0}\right)$ do not receive any tone, and thus they, have reserved the local link. All other nodes in the corresponding collision domains keep themselves at the quiet state.

Nodes $S_{0}$ and $H_{0}$ have reserved the corresponding local link simultaneously at the $(i+2)$-th slot. Both nodes send RTS control packets instead of data packets at the beginning of the $(i+3)$-th slot. The destination node, $R$, receives both control packets successfully due to the slight delay caused by the uncertainty of the acoustic channel. The destination node $R$ prioritizes the control packets based on their arrival time and gives higher priority to the packet that arrives at the latest. Then, it broadcasts a CTS packet at the beginning of the $(i+4)$-th slot. All nodes of the domain $h$ keep themselves in sleep mode, while the transmission of the data packet continues from node $S_{0}$ to node $R$. Thus, by incorporating a two-phase reservation mechanism, data packet collisions are avoided in multi-hop networks.

\section{Quantitative Analysis}

In this section, we analyse the channel utilization, end-to-end delay and energy consumption for the proposed protocol and evaluate its performance with the T-Lohi protocol by modelling the protocols in a typical sensor network scenario.

\subsection{Channel Utilization}

In order to analyse the performances of the proposed protocol for a multi-hop network with hidden collision domains, we assume the network topology utilized by Tobagi and Kleinrock [22]. The analysis is based on the following assumptions:

1. The network consists of a set of source nodes and a single receiving node. Each node is hidden from the rest of the nodes, but connected to the receiving node. We assume that there are $N+1$ nodes, including the receiving node. In this setup, each source node experiences $N-1$ hidden nodes. Each source node with a receiving node forms a collision domain.

2. The packet arrival rate to the MAC layer is assumed to have a Poisson distribution with a mean arrival rate of $\lambda$ packets/seconds.

3. Each data packet has a constant transmission duration of $T_{d}$ seconds.

4. The inter-arrival time between two consecutive data packets is exponentially distributed with a mean of $\frac{1}{\lambda}$ seconds.

5. Each sending node acts as a source, and the receiving node acts as a sink. 
The channel utilization, $C_{u}$ of the network, as defined in [22], is given by:

$$
C_{u}=\frac{\bar{U}}{\bar{B}+\bar{I}}
$$

where $\bar{U}$ represents the expected time duration that the receiving node spends for receiving the payload without collision, $\bar{I}$ represents the expected idle duration and $\bar{B}$ represents the expected duration of the busy period with or without collisions. At the receiving node, a collision could occur when a data packet collides with other data packets or with tones. With respect to the time-line of the receiving node, the expected vulnerability period for a data packet collision is equal to $T_{d}$, which is the transmission duration of the data packet. This case holds when all nodes are synchronized and transmit only at the beginning of a slot. A typical successful data packet reception and the vulnerability duration can be visualized by Figure 4.

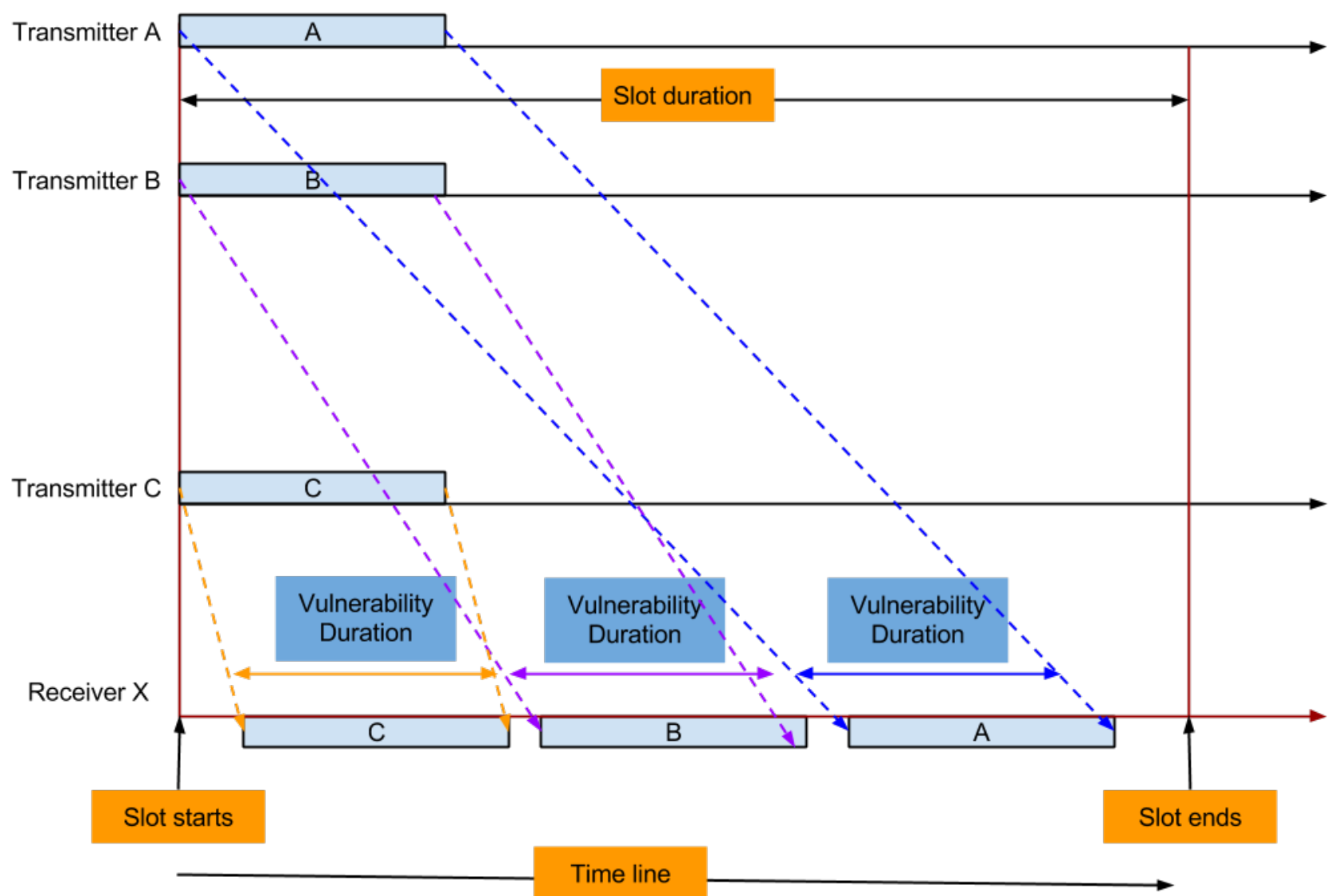

Figure 4. Successful reception and vulnerability duration of a packet.

After any successful local link reservation, the data packet transmission can be modelled as S-Aloha with non-negligible propagation delay. The expected vulnerability period for a packet will be $T_{d}$ for S-Aloha in an acoustic channel. Therefore, the probability of successful data transmission, $P_{s d}$, is given by:

$$
P_{s d}=e^{-T_{d} \lambda(N-1)}
$$


The total busy period can be divided into three parts:

- Busy due to successful data transmission, $T_{b s}$;

- Busy due to collisions of data packets at the receiver, $T_{b c}$;

- Time spent in local link reservation, $R_{e l}$.

The successful busy time is equal to the data transmission duration, $T_{b s}=T_{d}$. The busy time due to collisions is $T_{b c}=T_{d}+\tau_{\max }$, where $\tau_{\max }$ represents the maximum propagation time for any packet in the network. The expected number of $\mathrm{CR}$ required for a successful local link reservation, as calculated in [23], is given by:

$$
N_{C R}=\sum_{j=1}^{N_{c d}} P_{N_{c d}, j}\left(E\left[X_{N_{c d}}\right]+T_{j}\right)
$$

where $N_{c d}$ is the number of nodes of a collision domain (a single local node group) in which nodes are connected with one another by local links. In Equation (3), $E\left[X_{N_{c d}}\right]$ represents the expected length of a super round with $N_{c d}$. A super round consists of one or more contention rounds. A super round starts when nodes start contending in the current slot, and it ends when one or more nodes try to contend in any other future slot. The quantity, $P_{N_{c d}, j}$, represents the transition probability of the Markov chain used to model the channel reservation and $T_{j}$ represents the expected time of channel reservation of any node where the number of nodes in the local node group is $N_{c d}$ and $j$ represents the index of the nodes [23]. The mean idle time is the time that the sink node is not receiving any packets and is given by $\bar{I}=\frac{1}{N \lambda}$, and the mean useful data utilization time, $\bar{U}$, is given by $\bar{U}=P_{s d} T_{d} \alpha$, where $\alpha=\frac{\text { Pay Load Length }}{\text { Data Packet Length }}$. The local link reservation period calculated in Equation (3) is also affected by hidden collision domains. We assume that the local link reservation period of each collision domain is independent of each other and is uniformly distributed with the probability $\frac{1}{N-1}$, where $N-1$ represents the number of hidden collision domains. It should be noted that the number of hidden collision domains represents the number of hidden nodes, since from each collision domain, only one node is able to contend to control the hidden link. All other nodes are eliminated from contending through the local link reservation phase. A single contention round is equal to slot duration, $T_{s l}=T_{t}+\tau_{\max }$. Hence, the effective local link reservation period is given by:

$$
R_{e l}=N_{C R}(N-1) T_{s l}
$$

Since the T-Lohi protocol has similar channel reservation as local link reservation, the channel utilization for T-Lohi is given by:

$$
C_{u}=\frac{P_{s d} T_{d} \alpha}{P_{s d} T_{b s}+\left(1-P_{s d}\right) T_{b c}+R_{e l}+\frac{1}{N \lambda}}
$$

In the proposed protocol, the slot duration is increased to $T_{s l, p}=\tau_{\max }+\max \left(T_{N P}\right)$, where $T_{N P}$ represents the transmission duration of the notification packets. After a successful local link reservation, the sender and the receiver exchange notification packets to control the hidden links. The time duration of this exchange is $2 T_{s l, p}$. If the destination node successfully receives one or more RTS packets, it sends a CTS packet, which is successfully delivered to the sender in the next slot. The effective vulnerability duration for exchanging notification packets is $T_{R T S}+T_{C T S}$, where $T_{R T S}$ and $T_{C T S}$ represent the duration of the RTS and CTS packet, respectively. 
Hence, the probability of successful data transmission for the proposed protocol is calculated as follows:

$$
P_{s d, p}=e^{-\left(T_{R T S}+T_{C T S}\right) \lambda \beta(N-1)}
$$

In Equation (6), $\beta$ refers to the co-efficient of the channel access rate to start the contention for the hidden link reservation phase. Prior to the point of saturation of the network, the value of $\beta$ remains one, but when the network reaches saturation, $\beta$ follows the equation, $\beta=\frac{\lambda_{t h}}{\lambda}$, where $\lambda_{t h}$ is the packet arrival rate in the point of saturation. The time required for successful data transmission is $T_{b s, p}=2 T_{s l, p}+T_{d}+\tau_{\text {max }}$, and the time spent for the failed transmission or collision is $T_{b c, p}=2 T_{s l, p}$ [14]. The expected time required for the local link reservation is given by ([13]):

$$
R_{e l, p}=N_{C R}(N-1) T_{s l, p}
$$

The mean idle time is $\bar{I}=\frac{1}{N \lambda \beta}$, and the mean useful data utilization time is $\bar{U}=P_{s d, p} T_{d} \alpha$. Therefore, the channel utilization for the proposed protocol is given by ([22]):

$$
C_{u}=\frac{P_{s d, p} T_{d} \alpha}{P_{s d, p} T_{b s, p}+\left(1-P_{s d, p}\right) T_{b c, p}+R_{e l, p}+\frac{1}{N \lambda \beta}}
$$

\subsection{End-to-End Delay}

Previously, we have calculated the probability of a data packet successfully being delivered to the receiver without any collision. Here, we assume that each transmission cycle (local link reservation, hidden link control and data transmission) event is independent with respect to one another. Therefore, the event of successful transmissions preceded by zero or more unsuccessful transmission events can be modelled as a geometric random variable [22]. For T-Lohi, the expected number of transmissions required for a single successful data packet reception by a receiver is given by:

$$
\begin{aligned}
Q & =\sum_{i=0}^{\infty}\left(1-P_{s d}\right)^{i} P_{s d} \\
& =\frac{1-P_{s d}}{P_{s d}}
\end{aligned}
$$

Similarly, for the proposed protocol, the expected number of transmissions required for a single successful data packet reception is given by:

$$
\begin{aligned}
Q_{p} & =\sum_{i=0}^{\infty}\left(1-P_{s d, p}\right)^{i} P_{s d, p} \\
& =\frac{1-P_{s d, p}}{P_{s d, p}}
\end{aligned}
$$

where $P_{s d, p}$ and $P_{s d}$ are the probabilities of successful transmission for the proposed protocol and T-Lohi, respectively. In the case of the T-Lohi protocol, the total time of data transmission and propagation is $T_{d}+\tau_{\max }$ plus the local link reservation time, $R_{e l}$. The end-to-end delay is given by:

$$
D=Q\left(T_{d}+\tau_{\max }+R_{e l}\right)+T_{d}+\tau_{\max }+R_{e l}
$$

Similarly, the delay for the proposed protocol is given by:

$$
D_{p}=Q_{p}\left(2 T_{s l, p}+R_{e l, p}\right)+T_{d}+\tau_{\max }+2 T_{s l, p}+R_{e l, p}
$$




\subsection{Energy Consumption}

Assume that maximum transmission power is given by $W$ for tone, control and data packets. In the case of the T-Lohi protocol, energy loss due to data collision for a single successful data packet transmission is given by:

$$
E_{d c}=Q T_{d} W
$$

Energy is also wasted for channel reservations that ended with data collisions. The energy loss due to a failed reservation period is given by:

$$
E_{f r}=Q\left(\frac{R_{e l}}{T_{s l}}\right) T_{t} W
$$

The energy required for a successful reservation period, $E_{s r}$, and data transmission, $E_{s}$, is respectively given by:

$$
E_{s r}=\left(\frac{R_{e l}}{T_{s l}}\right) T_{t} W
$$

and:

$$
E_{s}=T_{d} W
$$

Hence, the total energy overhead or loss for a single successful data packet transmission for the T-Lohi protocol is given by:

$$
E_{l}=E_{d c}+E_{f r}+E_{s r}
$$

In the case of the proposed protocol, energy is lost due to notification packet collisions and failed local link reservations. Energy loss due to notification packet collisions is given by:

$$
E_{n p c}=Q_{p}\left(T_{C T S}+T_{R T S}\right) W
$$

while energy loss due to failed local link reservation is given by:

$$
E_{f r, p}=Q\left(\frac{R_{e l, p}}{T_{s l, p}}\right) T_{t} W
$$

The energy required for a successful local link reservation, $E_{s r, p}$, hidden link control, $E_{n p s}$, and data packet transmission, $E_{s, p}$, is respectively given by:

$$
\begin{gathered}
E_{s r, p}=\left(\frac{R_{e l, p}}{T_{s l, p}}\right) T_{t} W \\
E_{n p s}=\left(T_{C T S}+T_{R T S}\right) W
\end{gathered}
$$

and:

$$
E_{s, p}=T_{d} W
$$

Hence, total energy overhead or loss for a single successful data packet transmission for the proposed protocol is given by:

$$
E_{l, p}=E_{n p c}+E_{f r, p}+E_{s r, p}+E_{n p s}
$$




\subsection{Quantitative Evaluation}

The performances of the proposed protocol and the T-Lohi protocol are evaluated to demonstrate how the hidden collision domain and traffic loads can affect the channel utilization, the end-to-end delay and the energy overhead. The same parameters used in [9] are utilized to fairly compare the results. Table 1 provides the values of protocol parameters used in both quantitative analysis and simulation. The bit rate of the acoustic modems (wake-up tone and data receiver) and power consumption parameters are listed in Table 2.

Table 1. Protocol parameters. CTS, clear to send; RTS, request to send.

\begin{tabular}{cc}
\hline Parameters & Length (in Bytes) \\
\hline Payload & 625 \\
AppHeader & 5 \\
NetwHeader & 5 \\
MAC Header & 5 \\
PhyHeader & 5 \\
Tone & 5 \\
CTS & 10 \\
RTS & 10 \\
\hline
\end{tabular}

Table 2. Acoustic modem parameters.

\begin{tabular}{ccc}
\hline \multicolumn{3}{c}{ Bit-Rate: 8000 bps } \\
\hline Mode & Data & Wake-Up Tone \\
\hline Transmit (max) & $2 \mathrm{~W}$ & $2 \mathrm{~W}$ \\
Receive & $20 \mathrm{~mW}$ & $0.5 \mathrm{~mW}$ \\
Idle/Listen & $20 \mathrm{~mW}$ & $0.5 \mathrm{~mW}$ \\
\hline
\end{tabular}

Figure 5 demonstrates the effects of hidden nodes and packet arrival rate on the overall channel utilization of the network. The proposed protocol outperforms the T-Lohi protocol for all network configurations (networks of 1, 3 and 5 hidden nodes). At higher loads, especially when the network is overloaded, the difference between channel utilizations of the two protocols widens significantly. We observe that the channel utilization of the T-Lohi protocol is not stable, even at low loads when the number of hidden nodes is high. However, the channel utilization of the proposed protocol is stable and significantly outperforms that of the T-Lohi protocol. As an example, for the network configuration having three hidden nodes, the channel utilizations of the T-Lohi protocol and the proposed protocol are 0.004 and 0.19 , respectively, when the traffic rate is 2.0 packets per second. This shows the impact of hidden nodes in the T-Lohi protocol, which causes a drastic decrease in channel utilization. The channel utilization of the T-Lohi protocol under high traffic loads is low, and it degrades with an increasing number of hidden nodes, while the proposed protocol provides stable and better channel utilization under the same conditions. 


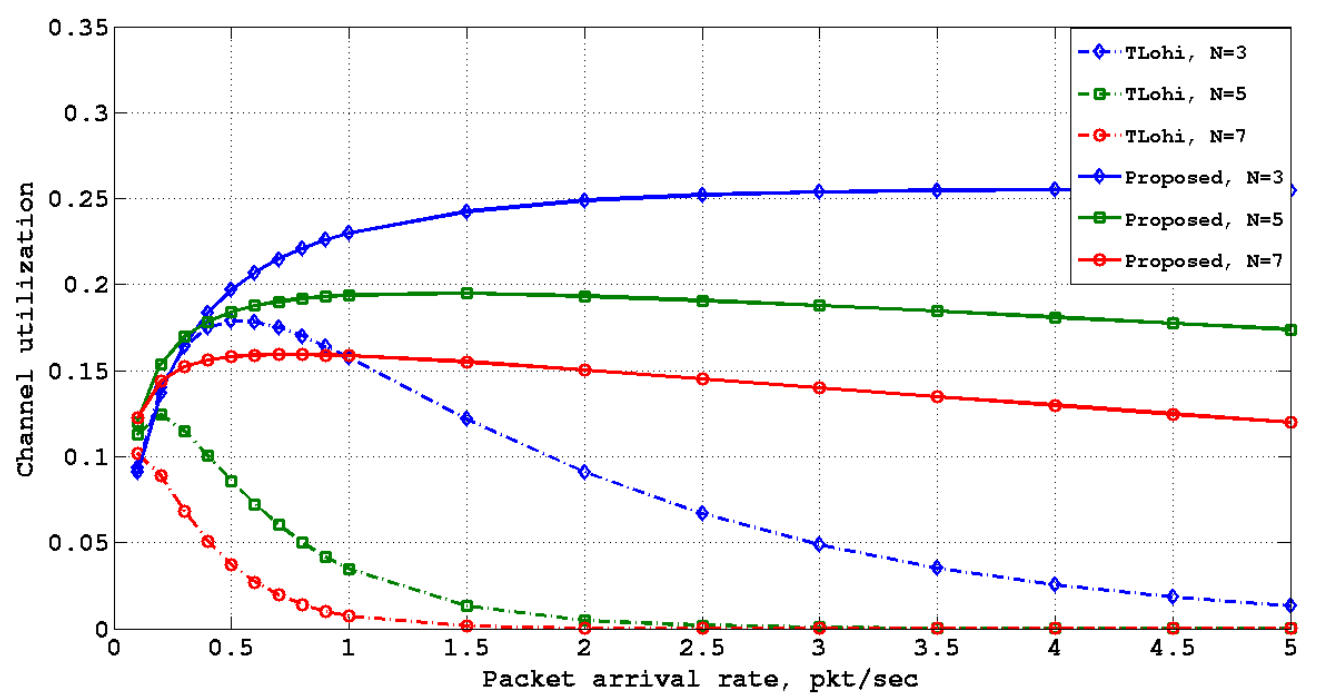

Figure 5. Channel utilization vs. packet arrival rate.

The proposed protocol achieves lower and more stable end-to-end delays than the T-Lohi protocol, as shown in Figure 6. As the number of hidden nodes and packet arrival rates increase, the end-to-end delay of the T-Lohi protocol increases by several orders of magnitude, whereas the proposed protocol achieves constant end-to-end delays. For the sake of illustration for the network configuration having three hidden nodes, end-to-end delays for the proposed protocol are 3.32 and $3.78 \mathrm{~s}$ considering packet arrival rates of two and five packets per second, respectively. However, under the same conditions, end-to-end delays for the T-Lohi protocol are extremely long, which is not acceptable for a viable network. This improvement is achieved by eliminating data packet collisions caused by hidden nodes.

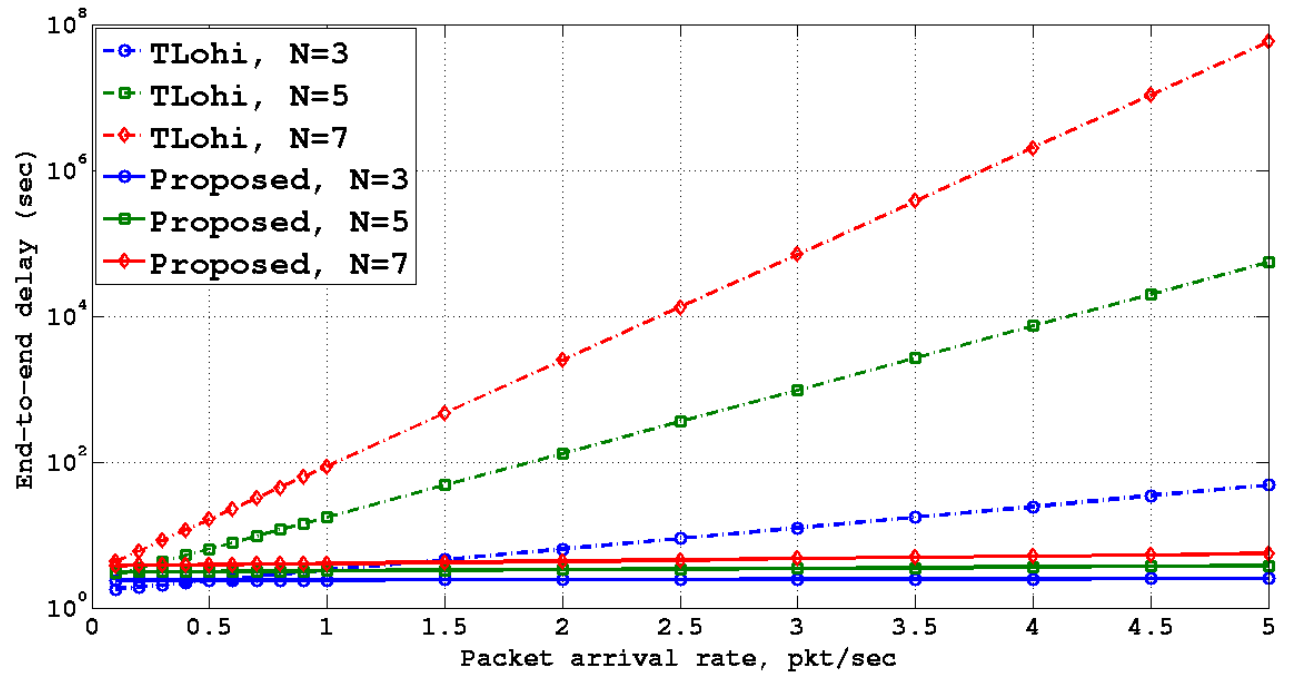

Figure 6. End-to-end delay vs. packet arrival rate.

In Figure 7, we have compared the total energy loss/overhead per single successful data packet transmission for both the T-Lohi protocol and the proposed protocols. From the performance graph, the proposed protocol outperforms the T-Lohi protocol irrespective of any traffic load and the number of hidden nodes in the network. Moreover, the proposed protocol shows stability and exhibits 
constant energy overhead in the saturation region, whereas the energy overhead of the T-Lohi protocol increases indiscriminately.

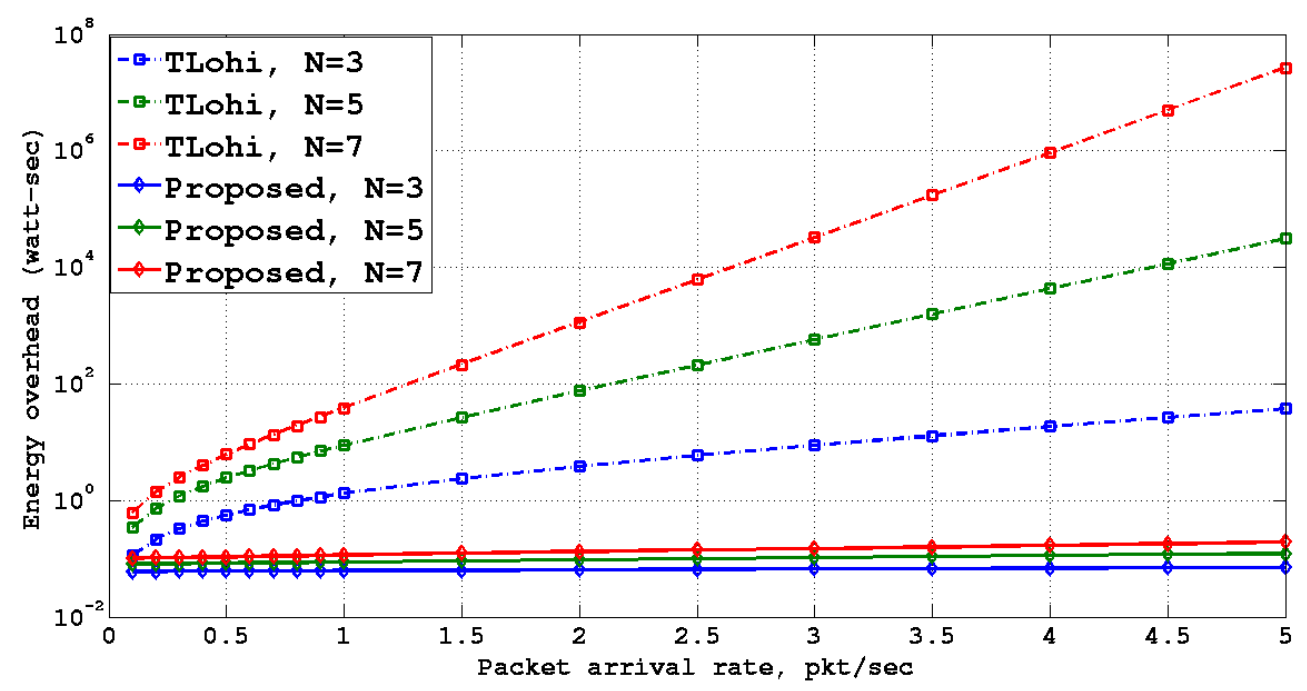

Figure 7. Energy overhead vs. packet arrival rate.

\section{Simulation Results}

In this section, both the proposed protocol and the T-Lohi protocol are simulated under similar conditions to demonstrate the effects of the improvement achieved with the proposed protocol. We also simulated the S-FAMA protocol and compared it to the proposed protocol. We have used OMNET++ [24] as the network simulation platform and modified the MiXiM [25,26] framework to make it suitable for UWASN. MiXiM is an OMNeT++ modelling framework created for mobile and fixed wireless networks (wireless sensor networks, body area networks, ad hoc networks, vehicular networks, etc.) and offers detailed models of radio wave propagation, interference estimation, radio transceiver power consumption and wireless MAC protocols (e.g., ZigBee) [27].

\subsection{Experimental Setup}

A star topology is adopted to demonstrate the effect of hidden nodes on the the proposed protocol and the T-Lohi protocol. The star topology is utilized in simulations to simplify and identify hidden nodes precisely. In our simulation setup, every node can communicate with the centre node (sink) and is hidden to all other nodes, which allows us to control the number of hidden nodes. Sensor nodes are deployed in a three-dimensional space whose height, width and depth is $1200 \mathrm{~m}$. The sink (centre) node is situated at the origin, and the source nodes are deployed on the $\mathrm{X}, \mathrm{Y}$ and $\mathrm{Z}$ axes. We have performed the simulation for different numbers of nodes in the network. The position of each node is uniformly distributed and bounded along the corresponding axis, such that each source node must be out of the interference range of other source nodes, as shown in Figure 8. The S-FAMA protocol has been simulated exploiting the same network topology and parameters in order to compare and evaluate the the proposed protocol with anRTS-CTS-based protocol, i.e., S-FAMA. 


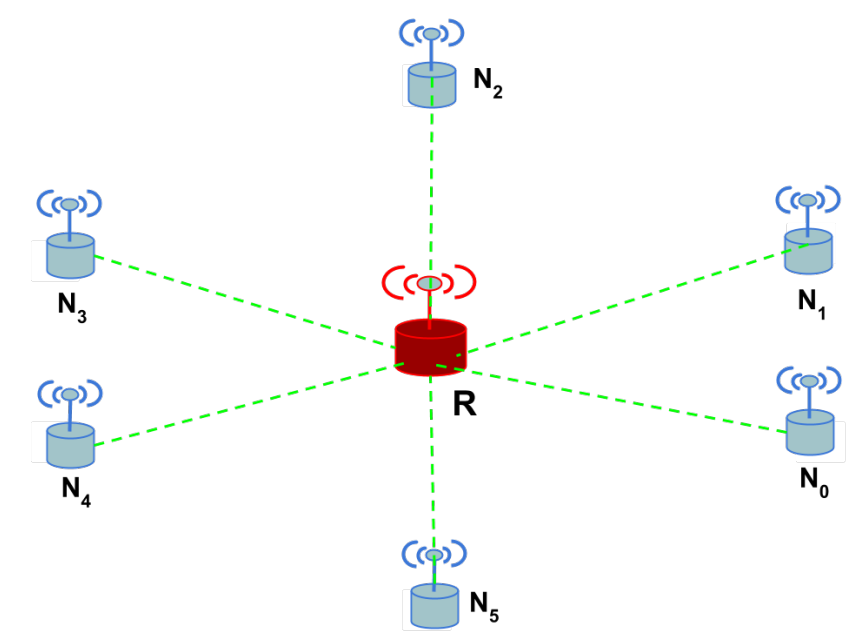

Figure 8. Network topology used for the simulation.

\subsection{Protocol Parameters}

In order to maintain a fair comparison between the two protocols (the proposed and T-Lohi protocols), we kept the values of the protocol parameters the same as [9]. The simulation time for each run is $500 \mathrm{~s}$. The payload size is 650 bytes, and the header length of each layer (from application to physical) is fixed to five bytes, while the length of the RTS and CTS packets is 10 bytes. Each node's application layer generates packets according to the Poisson distribution with a mean arrival time equal to $\frac{1}{\lambda}$. For each $\frac{1}{\lambda}$ value, 250 simulation runs are performed, and the average value of the runs is reported. The maximum transmission power of the acoustic modem is set to two Watts, and the maximum interference range is $500 \mathrm{~m}$. The acoustic channel path loss is calculated using Thorpe's model [28]. The acoustic modem data transmission rate is assumed to be 8000 bps. Furthermore, all nodes are assumed to be static during each simulation run. Protocol and acoustic modem parameters are listed in Tables 1 and 2, respectively.

\subsection{Results for the Single-Hop Star Topology}

Figure 9 shows the effect of hidden nodes on the channel utilization of the T-Lohi protocol. Solid lines in the graph represent the channel utilization of the T-Lohi protocol with respect to the offered load (number of packets/s), where all nodes in the network are connected with one another and packets are sent to a single receiving node, whereas dashed lines represent the channel utilization of the T-Lohi protocol for multi-hop networks with hidden nodes. Simulation results show that the T-Lohi protocol performs well when there are no hidden nodes; however, it suffers data packet losses when hidden nodes are present in the network. The performance of the T-Lohi protocol exhibits similar behaviour as Aloha where performance increases to a maximum peak and then drops sharply with increasing traffic load. This indicates instability in the protocol, even when the packet rate is as low as 0.5 packets/s. Moreover, it is important to note that having even one hidden node in the network degrades the peak channel utilization by 50 percent compared to the no hidden node scenario. Our observations indicate that this performance loss is due to data-data and tone-data collisions in the presence of hidden nodes in the network. 


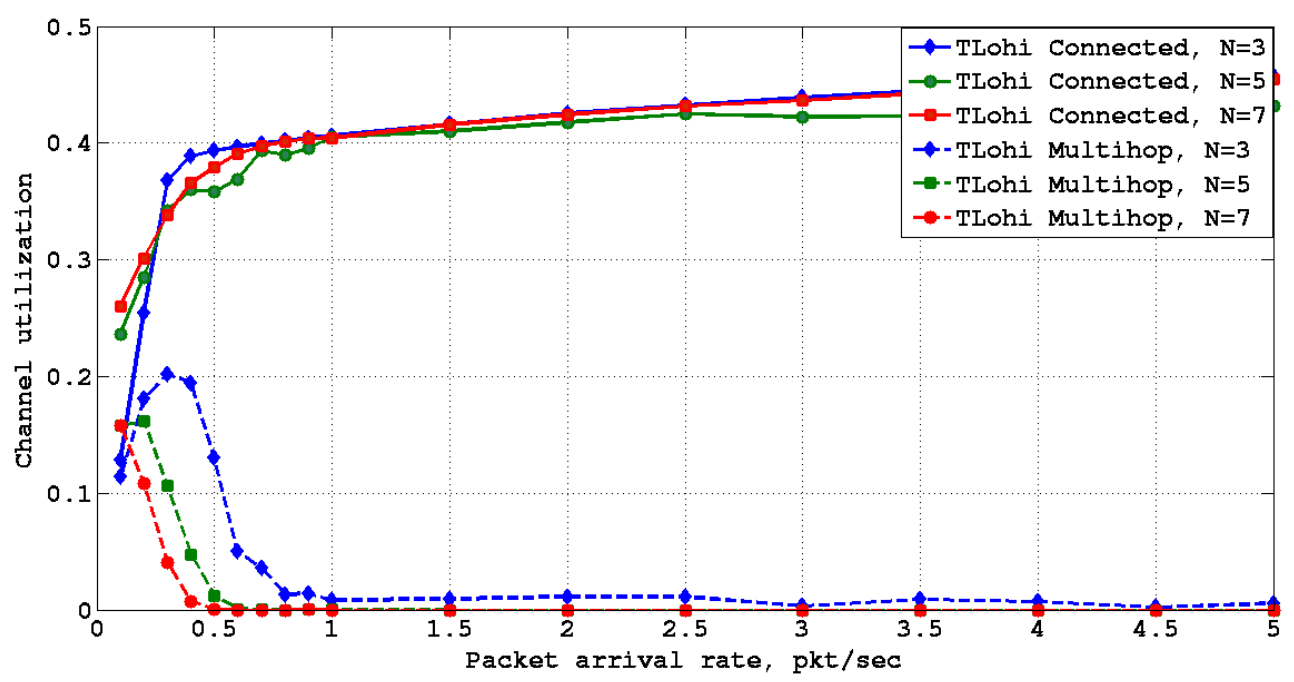

Figure 9. Effect of hidden nodes on T-Lohi MAC protocol.

Figure 10 shows the comparison of channel utilization between the proposed protocol and the T-Lohi protocol when there are hidden nodes present in multi-hop networks. Solid and dashed lines represent the proposed protocol and the T-Lohi protocol, respectively. First, the proposed protocol does not exhibit instability, and the performance does not decrease with increasing traffic load in the presence of hidden nodes. The peak channel utilization of the the proposed protocol is at least $50 \%$ more than that of the T-Lohi protocol. This is a clear indication that the proposed protocol successfully mitigates the impact of hidden nodes in the network using the hidden link control mechanism.

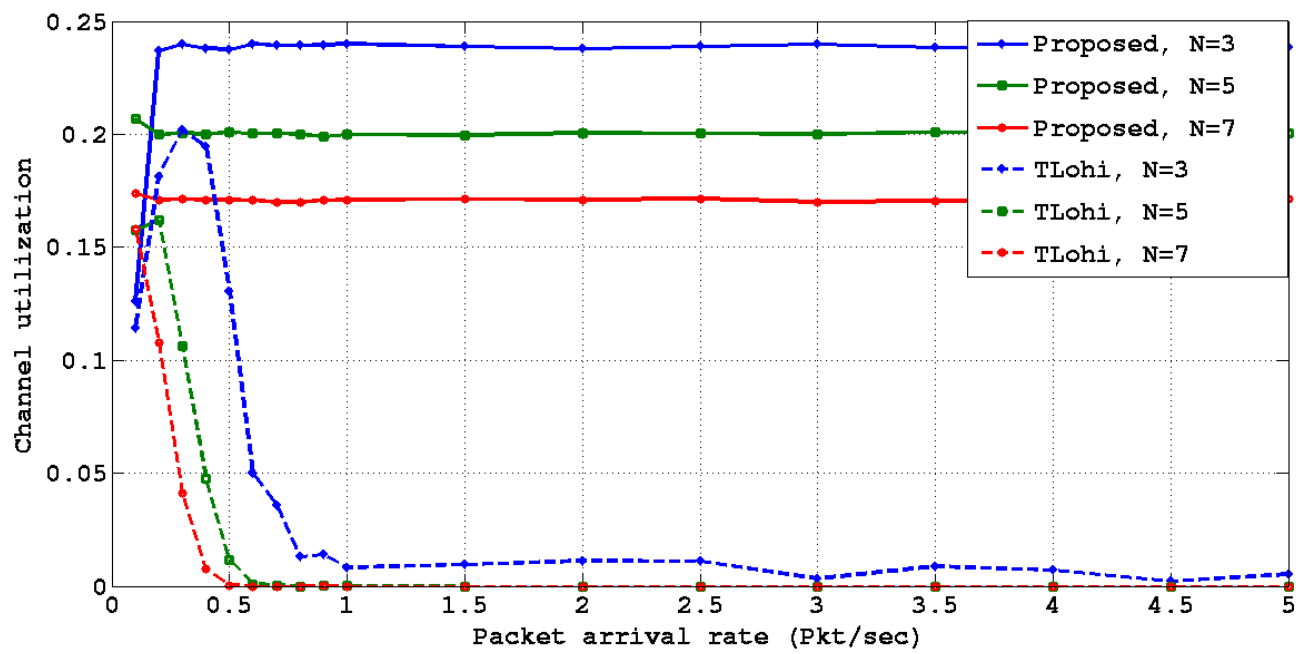

Figure 10. Comparison between T-Lohi and the proposed protocol.

Figure 11 depicts the comparative evaluation of channel utilization between the proposed protocol and the S-FAMA protocol. The proposed protocol shows better channel utilization as the number of hidden nodes increases. As the number of hidden nodes in the hidden collision domain increases, the probability of RTS/CTS collisions increases significantly since the potential transmitting node contends with the nodes from the local and hidden collision domains simultaneously. In contrast, the proposed protocol cuts off the contending nodes from the local collision domain in the local link reservation phase. 
Consequently, the collisions between notification packets in the second phase have been significantly reduced, which results in better channel utilization for the proposed protocol than the S-FAMA protocol.

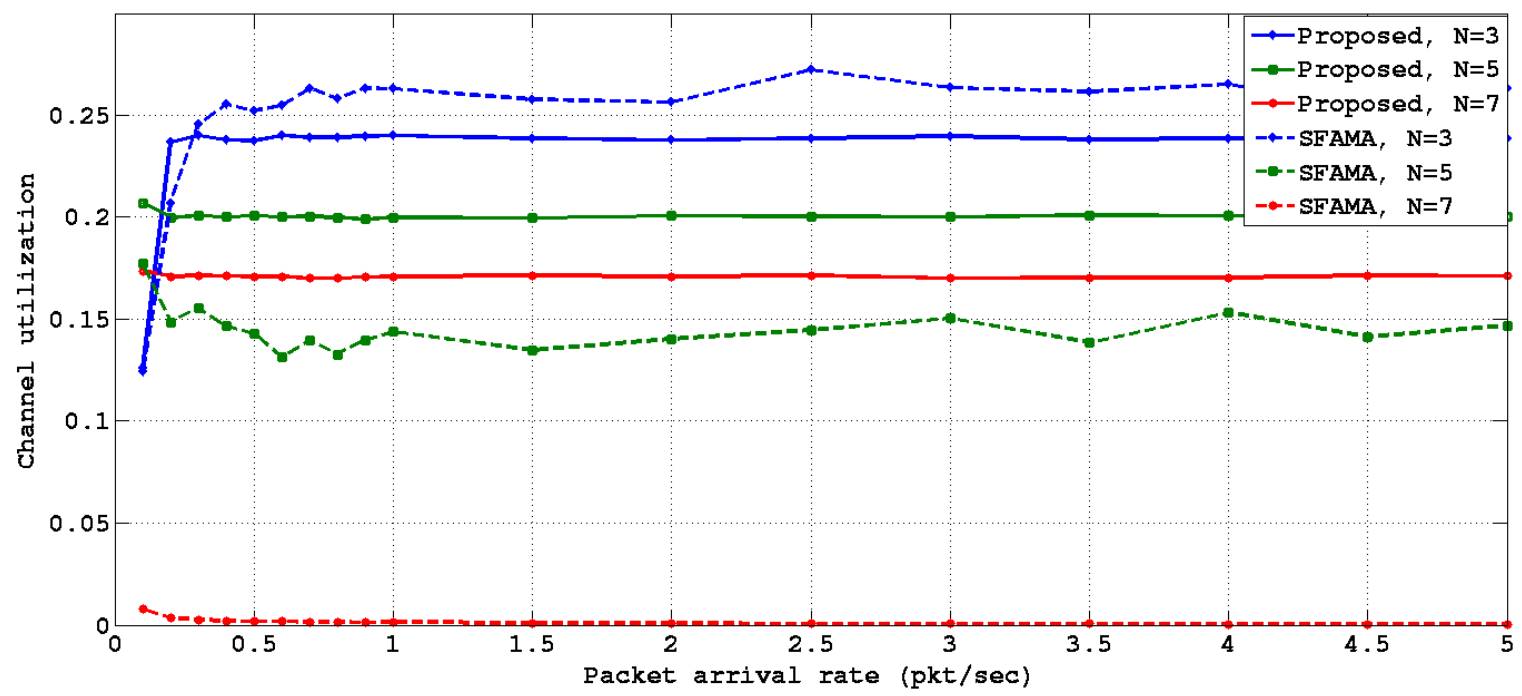

Figure 11. Comparison between slotted floor acquisition multiple access (S-FAMA) and the proposed protocol.

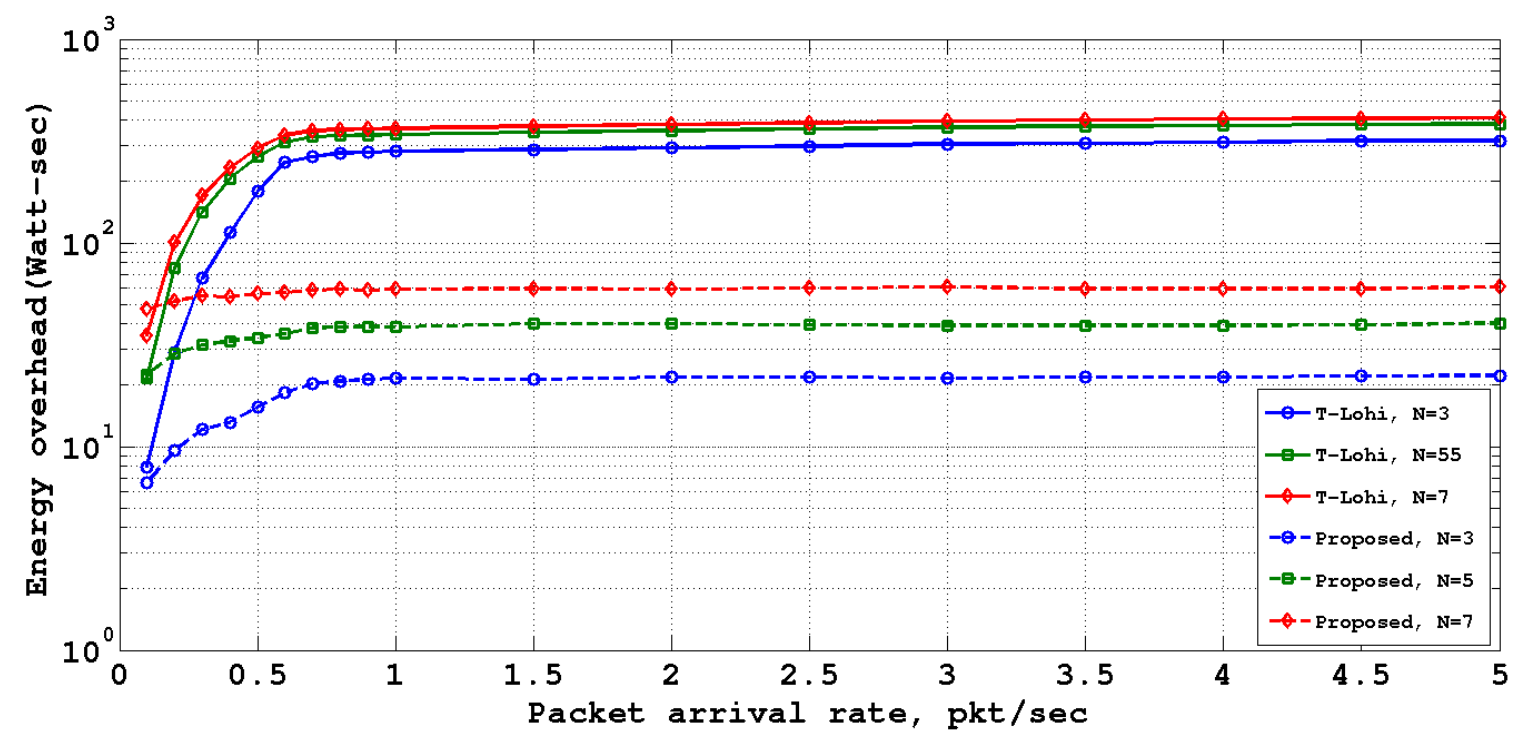

Figure 12. Comparison of energy overhead between T-Lohi and the proposed protocol.

The simulation outputs of energy overheads for the proposed protocol and the T-Lohi protocol are shown in Figure 12. As expected, the proposed protocol provides much better performance than the T-Lohi protocol. The energy overhead of the T-Lohi protocol is two orders of magnitude higher than the proposed protocol. Therefore, the proposed protocol works as an energy-efficient MAC protocol.

In Figures 13 and 14, comparisons of simulation and quantitative analysis results, e.g., channel utilization, are shown for both the proposed protocol and the T-Lohi protocol, respectively. From the graphs, we observe that there are no significant differences in trends between simulation results and those of the analytical results. The analytical results provide better performance, but simulation results represent more realistic performance. 


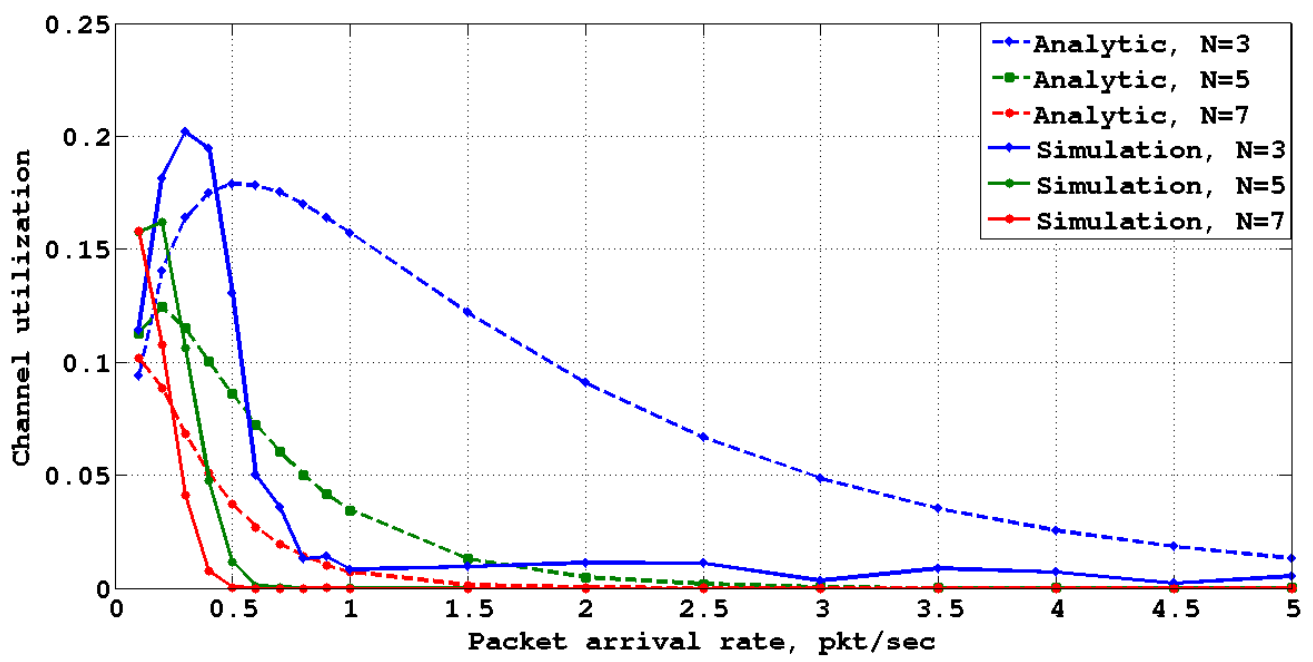

Figure 13. Simulation vs. quantitative analysis for T-Lohi.

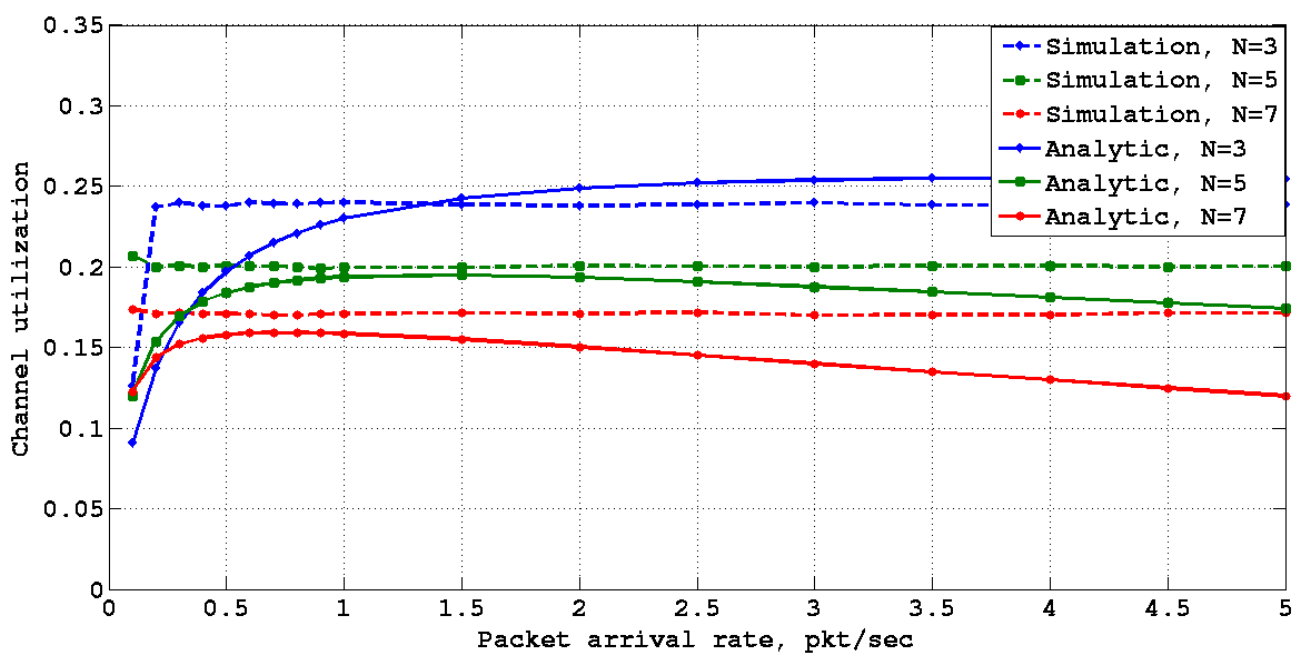

Figure 14. Simulation $v s$. quantitative analysis for the proposed protocol.

\subsection{Results for the Multi-hop Star Topology}

In order to simulate a multi-hop network, we have used a multi-hop star topology, where the nodes are distributed in a $4 \times 4$ kilometre area. The sink node is situated at the centre of the simulated area, and the source nodes are at the ends of the area. All other nodes between the sources and the sink work as relay nodes. We have simulated three different scenarios consisting of 2, 3 and 4 hops between the sources and the sink (i.e., 9, 13 and 17 nodes, respectively) in the network, but in each scenario, the number of source nodes are kept to four, as shown in Figure 15.

In each scenario, we have measured the mean packet success rate and end-to-end delay of the network. The packet success rate $\left(P_{s}\right)$ is defined as the ratio of the average number of successful data packets received by the sink node (in the application layer) to the average number of packets generated by all of the source nodes (in the application layer). End-to-end delay is defined as the ratio of the measured average end-to-end delay of the successfully received packets to the failure rate $\left(1-P_{s}\right)$ [22]. Actually, 
the success rate represents the probability of the successful reception of a data packet generated in the source. The measured end-to-end delay is the delay calculated at the sink when a data packet is successfully received by the sink. It is the time difference between packet generation time and the packet reception time. It is important to note that each iteration of the simulation has been run for $500 \mathrm{~s}$, and the mean has been calculated using 250 samples (iterations).

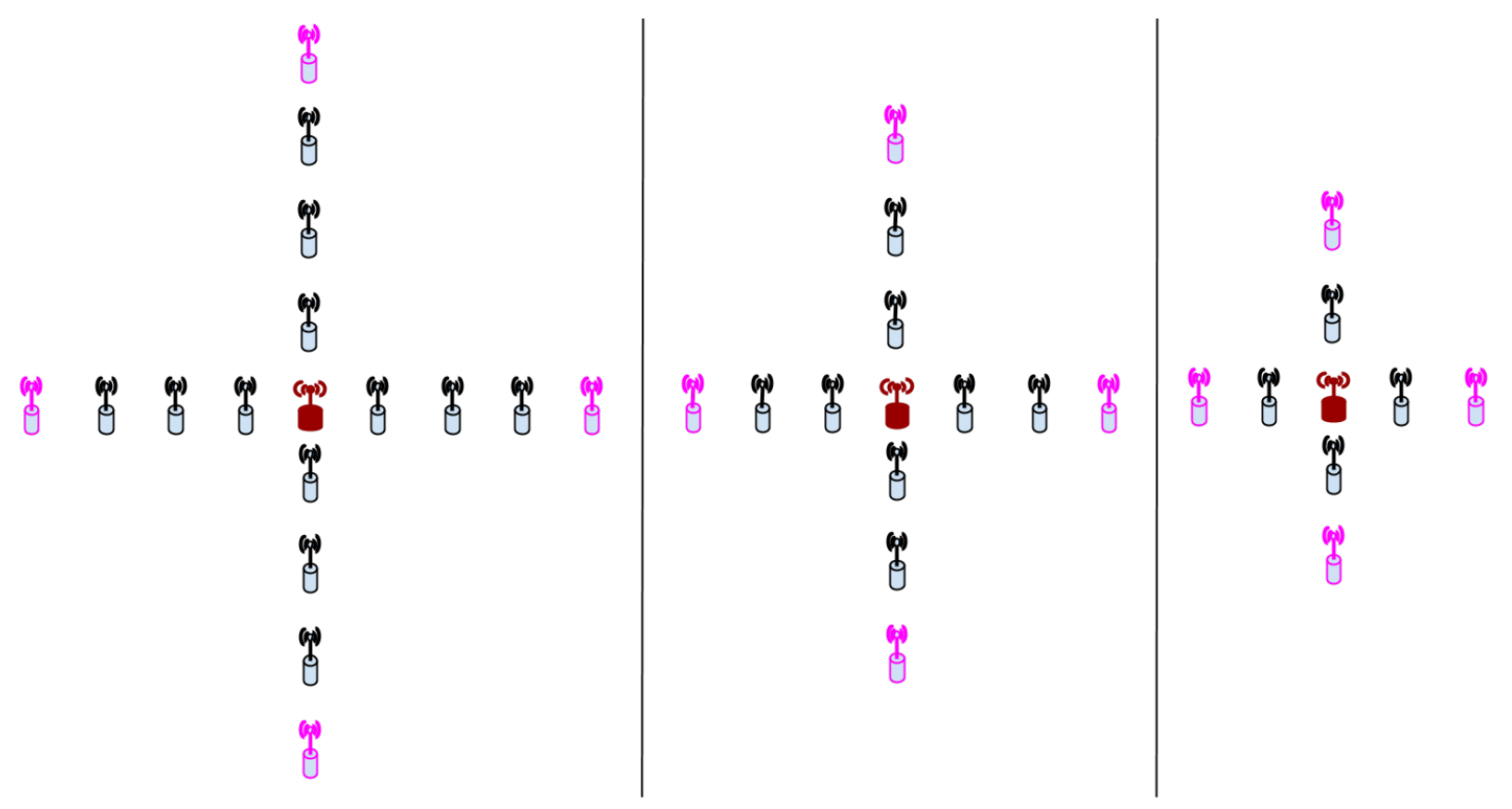

Figure 15. Multi-hop star topologies used for simulation.

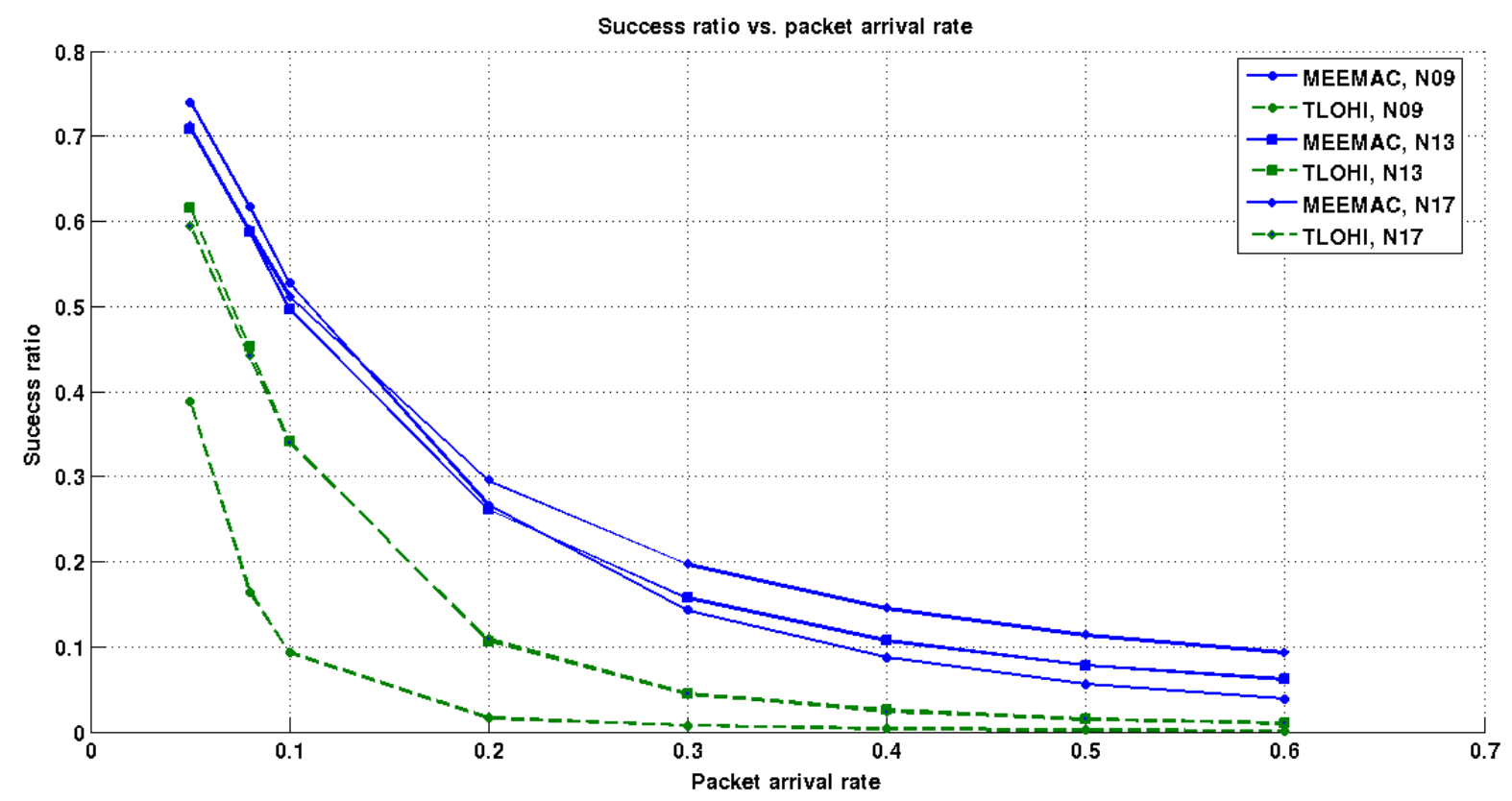

Figure 16. Success ratio (success probability) vs. the arrival rate for a multi-hop network. 


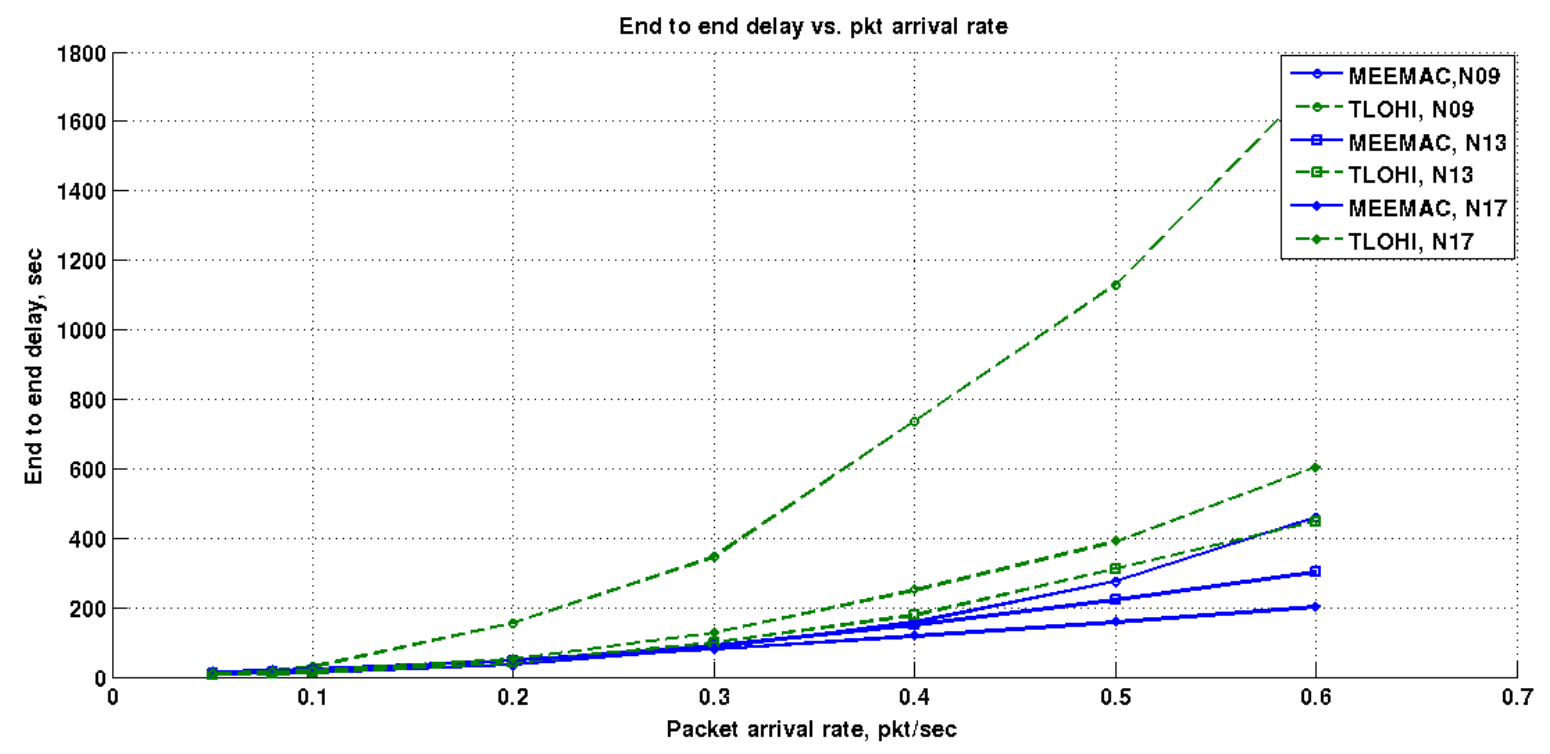

Figure 17. End to end delay vs. arrival rate for multi-hop network.

The success rates of the proposed protocol and the T-Lohi protocol have been depicted for different packet arrival rates in Figure 16. It is evident that as the packet arrival rate increases, the success rate for both protocols decreases; however, for the T-Lohi protocol, it decreases with a higher rate. Moreover, as the number of hops between the source and the sink increases, the effect of increasing hops adversely impacts the success rate of the T-Lohi protocol, while the proposed protocol demonstrates a very consistent and stable success rate.

Similarly, with respect to the end-to-end delay, the T-Lohi protocol experiences a couple orders of magnitude higher end-to-end delay compared to the proposed protocol. The corresponding graph is shown in Figure 17. Although both protocols undergo a similar pattern of increasing delay with respect to the increasing packet arrival rate, the T-Lohi protocol experiences unbounded delay.

By evaluating our proposed protocol through quantitative analysis and simulation, we find that the proposed protocol outperforms others in terms of energy efficiency, channel utilization and end-to-end delay. The proposed protocol achieves stable throughput even at high traffic load, while existing protocols become unstable and do not perform well. Besides that, the proposed protocol achieves better energy efficiency (at least one order of magnitude) and lower end-to-end delay (several orders of magnitude) compared to other existing protocols.

\subsection{Results for the Multi-Hop Topology with Multiple Sinks and Relays}

In order to evaluate the proposed protocol in a more generalized topology, simulations are done with a multi-hop network topology consisting of multiple sources, sinks and relay nodes in the same contention region, as shown in Figure 18.

$S_{1}, S_{2}, S_{3}, S_{4}, S_{5}$ and $S_{6}$ are source nodes and $D_{1}$ and $D_{2}$ are sink, as well as relay nodes. In that figure, $S_{1}, S_{2}$ and $D_{1}$ are in one contention region, $D_{1}, S_{5}, D_{2}$ and $S 6$ are in another contention region and, finally, $S_{3}, S_{4}$ and $D_{6}$ are in the other contention region. Data packets are transmitted from nodes $S_{1}$ and $S_{2}$ to node $D_{2}$ through node $D_{1}$, from node $S_{3}$ and $S_{4}$ to node $D_{1}$ through node $D_{2}$, from node 
$S_{5}$ to node $D_{1}$ and from node $S_{6}$ to node $D_{2}$. Simulation has been performed for different packet arrival rates with the proposed protocol and T-Lohi. The simulation result is shown in Figure 19.

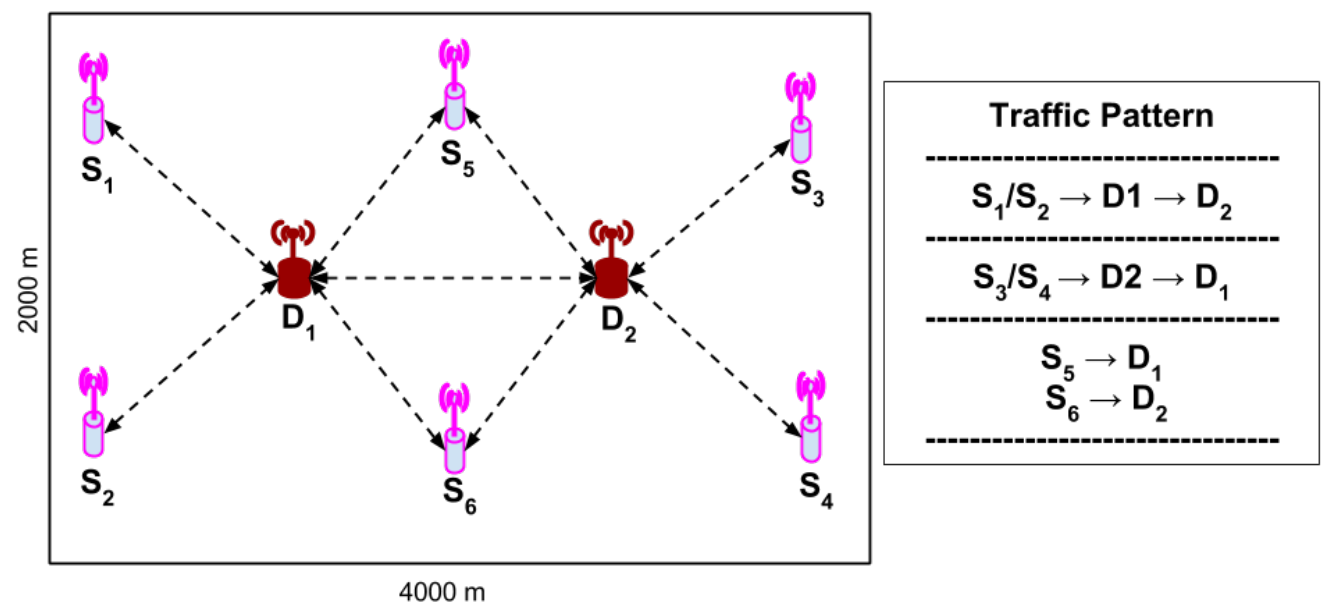

Figure 18. Multi-hop network with multiple sources, sinks and relays.

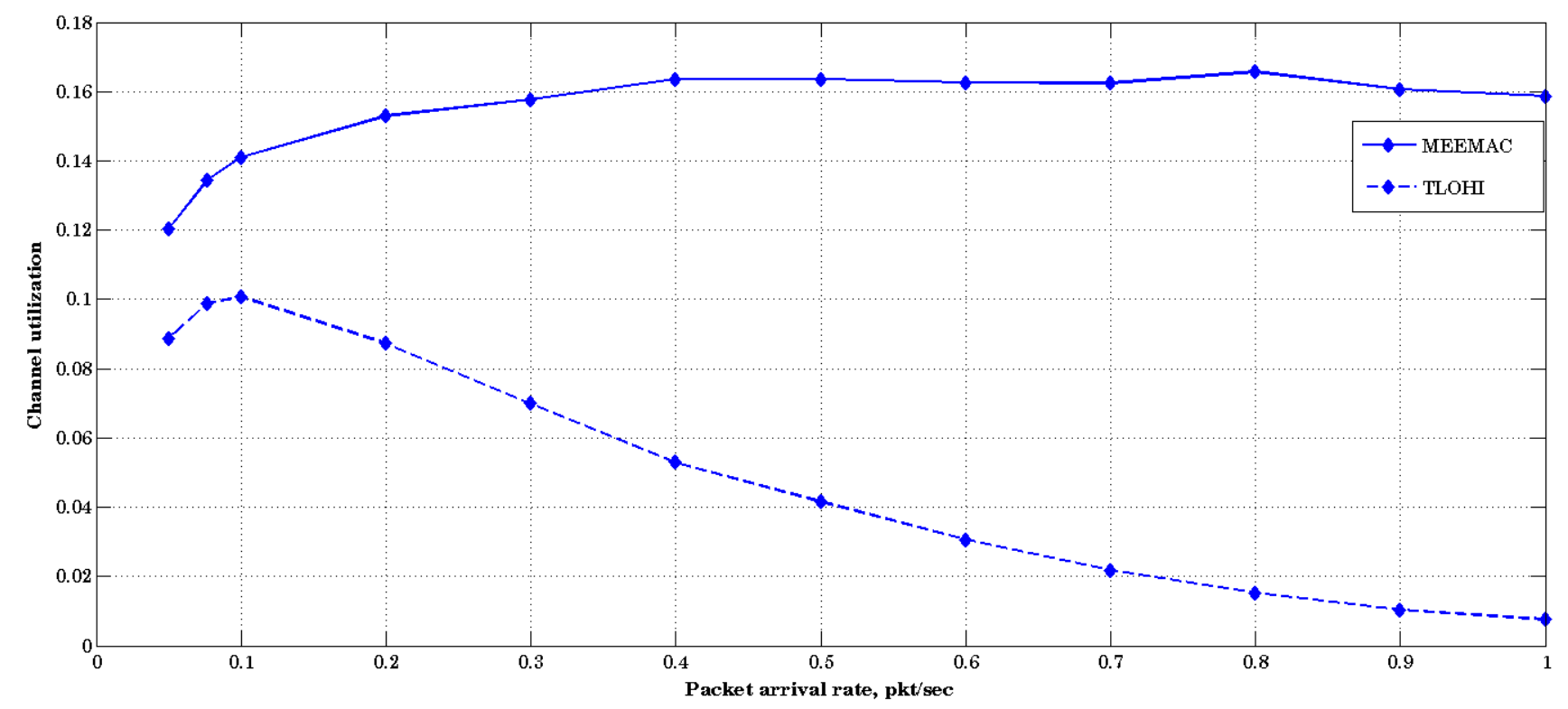

Figure 19. Channel utilization for T-Lohi and MEEMAC.

The results demonstrate that MEEMACoutperforms T-Lohi for all packet arrival rates. Moreover, MEEMAC shows stable channel utilization at higher packet arrival rates, while the channel utilization for T-Lohi decreases as the rate increases.

\section{Conclusions}

In this paper, a multi-hop-enabled energy-efficient MAC protocol for UWASN has been proposed, which exploits a novel two-phase contention resolution technique. A probabilistic model of the proposed protocol has also been developed to analyse the performance. The proposed protocol has been evaluated through quantitative analysis and simulations. The proposed protocol provides better 
energy efficiency, stability, higher channel utilization and lower end-to-end delay compared to the two existing MAC protocols for UWASN, namely the T-Lohi and the S-FAMA protocols. Results obtained from quantitative analysis and simulation have shown that the proposed MAC protocol has achieved at least twice as much channel utilization compared to the T-Lohi protocol under a high load and in the presence of hidden collision domains. Simulation results have shown that the proposed protocol reduced the energy lost at least by one order of magnitude and lowers the delay by several orders of magnitude compared to the T-Lohi protocol.

\section{Acknowledgements}

This work was supported in part by the NSERC Discovery Grant program (grant no. 262323-2011). The authors would like to thank the anonymous reviewers for their insightful comments.

\section{Author Contributions}

The work presented here was carried out in collaboration between all authors.

\section{Conflicts of Interest}

The authors declare no conflict of interest.

\section{References}

1. Rossing, T.D. Springer Handbook of Acoustics; Springer-Verlag: Berlin, Germany, 2007.

2. Akyildiz, I.F.; Pompili, D.; Melodia, T. State of the art in protocol research for underwater acoustic sensor networks. SIGMOBILE Mob. Comput. Commun. Rev. 2007, 11, 11-22.

3. Partan, J.; Levine, J.K.B.N. A Survey of Practical Issues in Underwater Networks. In Proceedings of the ACM WUWNet, Los Angeles, CA, USA, 29 September 2006; pp. 17-24.

4. Heidemann, J.; Ye, W.; Wills, J.; Syed, A.A.; Li, Y. Research Challenges and Applications for Underwater Sensor Networking. In Proceedings of the IEEE Wireless Communications and Networking Conference, Las Vegas, NV, USA, 3-6 April 2006; pp. 228-235.

5. Stojanovic, M. Acoustic (Underwater) Communications; John Wiley \& Sons, Inc.: Hoboken, NJ, USA, 2003.

6. Sozer, E.M.; Stojanovic, M.; Proakis, J.G. Underwater acoustic networks. IEEE J. Ocean. Eng. 2000, 25, 72-83.

7. IEEE Standard for Information Technology-Telecommunications and Information Exchange between Systems Local and Metropolitan Area Networks-Specific Requirements Part 11: Wireless LAN Medium Access Control (MAC) and Physical Layer (PHY) Specifications. IEEE Std 802.11-2012 (Revision of IEEE Std 802.11-2007). The IEEE Standards Association: Piscataway, NJ, USA; pp. 1-2793.

8. Syed, A.A.; Ye, W.; Heidemann, J.; Krishnamachari, B. Understanding spatio-temporal uncertainty in medium access with ALOHA protocols. In Proceedings of the Second Workshop on Underwater Networks (WuWNet '07), Montreal, QC, Canada, 9-14 September 2007; pp. 41-48. 
9. Syed, A.; Ye, W.; Heidemann, J. T-Lohi: A New Class of MAC Protocols for Underwater Acoustic Sensor Networks. In Proceedings of the 27th Conference on Computer Communications, Phoenix, AZ, USA, 13-18 April 2008; pp. 231-235.

10. Proakis, J.; Sozer, E.; Rice, J.; Stojanovic, M. Shallow water acoustic networks. IEEE Commun. Mag. 2001, 39, 114-119.

11. Rodoplu, V.; Park, M.K. An Energy-Efficient MAC Protocol for Underwater Wireless Acoustic Networks. In Proceedings of the MTS/IEEE OCEANS 2005, Washington, DC, USA, 17-23 September 2005.

12. Ye, W.; Heidemann, J.; Estrin, D. An energy-efficient MAC protocol for wireless sensor networks. In Proceedings of the Twenty-First Annual Joint Conference of the IEEE Computer and Communications Societies (INFOCOM 2002), New York, NY, USA, 23-27 June 2002; Volume 3, pp. 1567-1576.

13. Gibson, J.; Xie, G.; Xiao, Y.; Chen, H. Analyzing the Performance of Multi-Hop Underwater Acoustic Sensor Networks. In Proceedings of the OCEANS 2007-Europe, Aberdeen, UK, 18-21 June 2007; pp. 1-6.

14. Molins, M.; Stojanovic, M. Slotted FAMA: A MAC protocol for underwater acoustic networks. In Proceedings of the OCEANS 2006-Asia Pacific, Singapore, 16-19 May 2006; pp. 1-7.

15. Garcia-Luna-Aceves, J.J.; Fullmer, C.L. Floor acquisition multiple access (FAMA) in single-channel wireless networks. Mob. Netw. Appl. 1999, 4, 157-174.

16. Fullmer, C.L.; Garcia-Luna-Aceves, J.J. Floor Acquisition Multiple Access (FAMA) for Packet-Radio Networks. In Proceedings of the Conference on Applications, Technologies, Architectures, and Protocols for Computer Communication (SIGCOMM '95), Cambridge, MA, USA, 28 August-1 September 1995; pp. 262-273.

17. Bharghavan, V. MACAW: A Media Access Protocol for Wireless LANs. In Proceedings of the ACM SIGCOMM'94 Conference, London, UK, 31 August-2 September 1994; pp. 212-225.

18. WHOI. Acoustic Micro-Modem Overview, 2006-2013. Available online: http://acomms.whoi.edu/micro-modem/ (accessed on 24 August 2015).

19. Hong, L.; Hong, F.; Guo, Z.; Li, Z. ECS: Efficient Communication Scheduling for Underwater Sensor Networks. Sensors 2011, 11, 2920-2938.

20. Lee, J.W.; Cho, H.S. Cascading Multi-Hop Reservation and Transmission in Underwater Acoustic Sensor Networks. Sensors 2014, 14, 18390-18409.

21. Noh, Y.; Lee, U.; Han, S.; Wang, P.; Torres, D.; Kim, J.; Gerla, M. DOTS: A Propagation Delay-Aware Opportunistic MAC Protocol for Mobile Underwater Networks. IEEE Trans. Mob. Comput. 2014, 13, 766-782.

22. Tobagi, F.; Kleinrock, L. Packet Switching in Radio Channels: Part II-The Hidden Terminal Problem in Carrier Sense Multiple-Access and the Busy-Tone Solution. IEEE Trans. Commun. 1975, 23, 1417-1433.

23. Syed, A.A.; Heidemann, J. Contention analysis of MAC protocols that count. In Proceedings of the Fifth ACM International Workshop on UnderWater Networks (WUWNet '10), Woods Hole, MA, USA, 30 September-1 October 2010; pp. 2:1-2:8. 
24. Varga, A.; Hornig, R. An Overview of the OMNeT++ Simulation Environment. In Proceedings of the 1st International Conference on Simulation Tools and Techniques for Communications, Networks and Systems \& Workshops (Simutools '08), Marseille, France, 3-7 March 2008; pp. 60:1-60:10.

25. Köpke, A.; Swigulski, M.; Wessel, K.; Willkomm, D.; Haneveld, P.T.K.; Parker, T.E.V.; Visser, O.W.; Lichte, H.S.; Valentin, S. Simulating wireless and mobile networks in OMNeT++ the MiXiM vision. In Proceedings of the 1st International Conference on Simulation Tools and Techniques for Communications, Networks and Systems \& Workshops, (Simutools '08), Marseille, France, 3-7 March 2008; pp. 71:1-71:8.

26. Wessel, K.; Swigulski, M.; Köpke, A.; Willkomm, D. MiXiM: The physical layer an architecture overview. In Proceedings of the 2nd International Conference on Simulation Tools and Techniques (Simutools '09), Rome, Italy, 2-6 March 2009; pp. 78:1-78:8.

27. Viklund, A. MiXiM: A simulator for wireless and mobile networks using the OMNeT++ simulation engine, 2007-2013. Available online: http://mixim.sourceforge.net (accessed on 24 August 2015).

28. Brekhovskikh, L.; Lysanov, Y. Fundamentals of Ocean Acoustics; Springer Series in Electrophysics; Springer: Berlin, Germany, 1982.

(c) 2015 by the authors; licensee MDPI, Basel, Switzerland. This article is an open access article distributed under the terms and conditions of the Creative Commons Attribution license (http://creativecommons.org/licenses/by/4.0/). 